\title{
Completando lo inacabado. Consideraciones sobre EL COMPOSITOR, EL INTÉRPRETE Y LA ORNAMENTACIÓN A TRAVÉS de los manuscritos de Johann Georg Pisendel \\ Completing the unfinished. Aproaches on the composer, THE PERFORMER AND THE ORNAMENTATION through the manuscripts of Johann Georg Pisendel
}

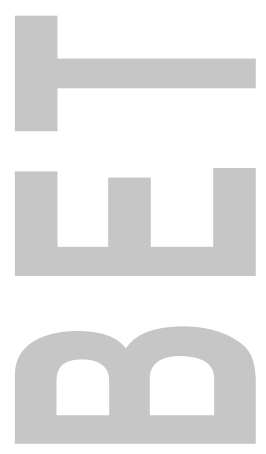

Francisco Javier Lupiáñez Ruiz•

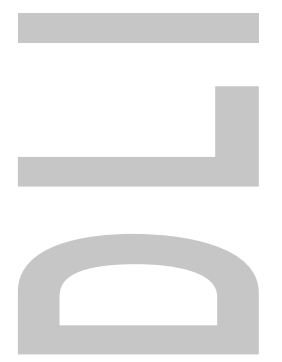

\section{RESUMEN}

El archivo conocido como Schrank II de la Sächsische Landesbibliothek - Staatsund Universitätsbibliothek. Dresden contiene gran parte de la inmensa colección personal de música instrumental que Pisendel se ocupó de reunir durante casi toda su vida.

En el archivo se encuentran los manuscritos para uso personal que contienen las anotaciones de Pisendel para su trabajo diario como intérprete y compositor. Un raro tesoro, ya que este tipo de manuscritos no estaban destinados a ser conservados después de su uso.

De especial interés son los manuscritos de sus propias composiciones. Entre los tachones y enmiendas compositivas encontramos también multitud de anotaciones destinadas a la interpretación, entre las que destacan las que servían para la ornamentación. Estos «manuscritos de trabajo» conviven en ocasiones con copias a limpio, el estudio combinado de estas fuentes arroja una interesante y novedosa visión de las múltiples facetas de Pisendel como compositor e interprete.

Este legado se hace eco de la práctica interpretativa de su época y nos invita a replantearnos de nuevo consideraciones básicas de la interpretación histórica como el papel del compositor y del intérprete así como a expandir los conceptos de composición, improvisación y ornamentación, dando a esta última un papel fundamental en la elaboración final de la obra musical.

- Javier Lupiáñez es musicólogo, violinista historicista y fundador de los ensembles Scaramuccia y Les Esprits Animaux y del sello discográfico y editorial Snakewood Editions. Como investigador ha publicado sobre ornamentación, Pisendel y Vivaldi, temas en los que se centra su tesis doctoral.

Recepción del artículo: 26-VI-2020. Aceptación del artículo: 29-VII-2020. 
Palabras clave: Interpretación histórica; composición; compositor; intérprete; improvisación; ornamentación; Pisendel.

\section{Abstract}

The archive known as Schrank II from the Sächsische Landesbibliothek - Staats- und Universitätsbibliothek Dresden holds much of the vast personal collection of instrumental music of Pisendel.

The archive contains manuscripts for personal use that include Pisendel's notes for his daily work as a performer and composer. This is a rare treasure, as these types of manuscripts were not intended to be preserved after its use.

Of special interest are the manuscripts of his own compositions. Among the compositional amendments we also find annotations intended for performance, among which those used for ornamentation stand out. These "working manuscripts", sometimes coexist with clean copies, the combined study of these sources yields an interesting and novel vision of the multiple facets of Pisendel as a composer and performer.

This legacy echoes the performance practice of his time and invites us to rethink basic considerations of historical interpretation such as the role of the composer and the performer as well as to expand the concepts of composition, improvisation and ornamentation, giving the latter a fundamental role in the final elaboration of the musical work.

Key words: HIP; composition; composer; performer; improvisation; ornamentation; Pisendel.

\section{INTRODUCCIÓN}

\section{I.1. Sobre el compositor, el intérprete y la ornamentación en el siglo XVIII}

«"No, no tengo nada que decir, yo solo soy un intérprete." “¿En oposición a qué?” pregunta el entrevistador, a lo que Leonhardt responde "a un músico de verdad, un compositor"»".

A mediados de los noventa uno de los pioneros de la música antigua, Gustav Leonhardt, manifestaba así su ideal romántico sobre el compositor, situándolo en un lugar que distaba mucho al que realmente ocupaba en el siglo XviII. El encumbramiento de la figura del compositor como máximo

${ }^{1}$ No, I have notbing to say, I am only a player.' 'As opposed to?' asks the interviewer, Leonhardt's response being 'to a real musician, which is a composer'. Traducción del autor. Si no se indicase lo contrario todas las traducciones son del autor.

Bernard David Sherman, Inside Early Music (Nueva York: Oxford University Press, 1997), 203-204. 
exponente de la creación musical es una idea surgida a principios del $\operatorname{XIX}^{2}$ y totalmente ajena a músicos como Bach o Couperin.

Cabe preguntarse, sobre todo entre los actuales especialistas en la interpretación históricamente informada, si aún se mantiene la actitud de Leonhardt ante la figura del compositor. Hace algunos años, Bruce Haynes afirmaba que aún hoy «los modernos intérpretes HIP3 están bajo el hechizo de estos cánones, $[\ldots]$ tomando la partitura como un ente intocable o inmutable» ${ }^{4}$.

Sin embargo la situación era muy diferente en el siglo xviII y la partitura recién salida de la pluma del compositor estaba muy lejos de ser una creación inmutable, de hecho, aún le faltaba un largo camino para considerarse como una obra acabada.

Antes de finales del siglo xvIII, los procedimientos de publicación reflejaban un contexto dominado por conceptos funcionales de la música basados en la interpretación. Se puede encontrar evidencia de esto en la falta de una distinción clara tanto en teoría como en la práctica entre la obra y la interpretación. Hoy, normalmente fechamos las composiciones de acuerdo a cuándo se completa el proceso de escritura, en el siglo xviII las composiciones para ocasiones públicas a menudo se fechaban de acuerdo con el momento de la actuación, e incluso si el músico comenzaba a ver la composición como una actividad que tenía lugar independientemente de la actuación, la mayoría todavía veía lo primero completarse realmente solo en lo último. ${ }^{5}$

La composición no podía estar completa sin la interpretación. Esto nos da una visión un tanto diferente a la de Leonhardt y nos lleva a pensar que a día de hoy parece claro, al menos en el

${ }^{2}$ Para un estudio más detallado sobre el concepto de obra musical en el siglo XVIII véase Michael Talbot, «The Work-Concept and Composer-Centredness», en The Musical Work: Reality or Invention?, ed. por Michael Talbot (Liverpool: Liverpool University Press, 2000).

${ }^{3}$ Siglas en inglés de Historically Inspired Performer. Traducido como «interpretación históricamente inspirada» es el término usado por Bruce Hynes en The End of Early Music (Nueva York: Oxford University Press, 2007). Haynes usa conscientemente el término «inspirada» y no «informada» como comúnmente suelen traducirse estas siglas.

${ }^{4}$ Modern HIP musicians are under the Canonic spell too. Usually Classically trained, they sometimes find themselves confusing fidelity to a style with fidelity to particular hero-composers. Against their own logic, they sometimes treat scores as untouchable (that is, unchangeable).

Bruce Haynes, The End of Early Music (Nueva York: Oxford University Press, 2007), 6.

${ }^{5}$ Before the end of the eighteenth century, publication procedures reflected a context dominated by a functional performance-based concepts of music. Evidence for these can be found in the lack of a clear distinction between work and performance that was pervasive both in practice and in terminology. Today, we normally date compositions according to when the process of writing is completed. In the 18th century, compositions for public occasions were often dated according to the time of performance, and even if musician were beginning to see composition as an activity that took place independently of performance, most still saw the former and truly completely only in the latter.

Friedemann Sallis, Music Sketches (Cambridge: Cambridge University Press, 2015), 240. 
ámbito académico, que el intérprete no solo tiene mucho que decir sino que, desde un punto de vista históricamente informado, su aportación es fundamental. ${ }^{6}$

Antes del siglo xix la «fidelidad a la partitura» ${ }^{7}$ significaba que se esperaba de los intérpretes que «completaran» ${ }^{8}$ la composición por sus propios medios en un contexto en el que el papel del compositor era mucho menos importante a lo que hoy estamos acostumbrados. El profesor Michael Talbot emplea un convincente símil para explicar el lugar que ocupaba el compositor en aquellos tiempos: «La coreografía, una práctica creativa en una esfera diferente, hoy ocupa un pequeño nicho en la conciencia pública muy comparable al de la composición musical hace trescientos años»?.

En este mismo artículo Talbot propone además un cambio de paradigma en cuanto a la concepción de obra musical en la primera mitad del siglo xviII. Rompiendo con el concepto de obra musical como un ente autónomo y dándole un carácter más pragmático. Una vez más vemos como ni la obra ni su creador disfrutan de la importancia que se les dará más tarde. El artículo es parte de un volumen editado por el propio Talbot cuyo título, The Musical Work: Reality or Invention? ${ }^{10}$ es ya de por sí bastante sugestivo y que se inspira en las ideas postuladas por el clásico de Lydia Goehr. ${ }^{11}$

En contraposición al estatus del compositor encontramos con que el intérprete gozaba de un papel predominante en la escena musical del siglo XVIII. Surgen en Europa genuinas estrellas de ópera como Farinelli o Cuzzoni o famosos violinistas como Veracini o Corelli. Los mejores artistas de su tiempo eran capaces de forjar una «reputación permanente, más allá de las obras que elegían interpretar. Una parte intrínseca de la identidad de la estrella fue la forma en que estamparon una personalidad en la conciencia del público» ${ }^{12}$. Esta personalidad se veía reflejada en el estilo interpretativo que hacía único a cada intérprete.

${ }^{6}$ Corey Jamason, «The Performer and the Composen», en The Cambridge History of Musical Performance, ed. por Colin Lawson y Robin Stowell (Cambrigde: Cambridge University Press, 2002), 105-34.

${ }^{7}$ John Butt, «Negotiating between work, composer and performer: rewriting the story of notational progress», en Playing with History: The Historical Approach to Musical Performance (Cambridge: Cambridge University Press, 2012), 96-122.

${ }^{8}$ Butt, «Negotiating between work...», 96-122.

${ }^{9}$ Choreography, a creative practice in a different sphere, today occupies a small niche in public awareness closely comparable with that of musical composition three hundred years ago.

Talbot, «The Work-Concept...», 174.

${ }^{10}$ Michael Talbot, ed., The Musical Work: Reality or Invention? (Liverpool: Liverpool University Press, 2000).

${ }^{11}$ Lydia Goehr, The Imaginary Museum of Musical Works: An Essay in the Philosophy of Music (Oxford: Claredon Press, 1992).

${ }^{12}$ Such top performers could establish a permanent reputation above and beyond the works they chose to perform. An intrinsic part of the star's identity was the way in which they stamped a performing personality on public consciousness. Simon McVeigh, «Performance in the 'Long Eighteenth Century': an Overview», en The Cambridge History of Musical Performance, ed. por Colin Lawson y Robin Stowell (Cambridge: Cambridge University Press, 2012), 490. 
Teniendo esto en cuenta es fácil comprender la gran cantidad de intervenciones o modificaciones que vemos en el repertorio de esta época. «La composición, el arreglo y la adaptación apenas se distinguen. Por el contrario, los tres se integraron en el proceso de preparación de actuaciones» ${ }^{13}$.

No cabe duda de que dentro de estas intervenciones del intérprete en la composición, la ornamentación juega un papel crucial. La gran presencia de la ornamentación como tema de estudio en los tratados impresos de esta época nos da una idea de su importancia. De hecho, estos tratados son una parte fundacional en la actual práctica historicista. Aunque ya en 1967 Frederick Neumann nos ponía sobre aviso sobre algunas limitaciones que podían derivarse del estudio de estos tratados, como la «simplificación pedagógica excesiva» que normalmente se encuentra en este tipo de trabajos de naturaleza didáctica. ${ }^{14}$ Esto llevó a Neumann a alentar a aquellos que buscan información sobre la interpretación históricamente informada en estas fuentes a hacerse una pregunta importante: «iA quién va dirigido el libro: a principiantes, estudiantes avanzados o artistas?» ${ }^{15}$.

Pero podemos ir un poco más allá y preguntarnos cómo sería la interpretación de esas estrellas de la escena barroca y si tendría mucho que ver con lo que encontramos en estos tratados destinados en su mayoría a un público general.

Visto desde esta perspectiva, los tratados impresos solo pueden ofrecer información limitada sobre la interpretación musical del siglo xvıII y para obtener una imagen más completa es necesario buscar otras fuentes, entre las cuales hay que destacar las fuentes manuscritas destinadas al uso privado de los propios músicos. ${ }^{16}$ Debido a su naturaleza efímera, estos documentos forman un raro corpus entre el conjunto de manuscritos que han sobrevivido hasta nuestros días, especialmente en comparación con el legado del siglo xix y xx. ${ }^{17}$ Sin lugar a dudas uno de los archivos más interesantes en relación a este tipo de manuscritos es el dejado por Johann Georg Pisendel. La colección personal de Pisendel reúne un inmenso registro de su actividad como coleccionista de música, alumno, profesor, compositor, intérprete o arreglista. Es difícil

\footnotetext{
${ }^{13}$ Composition, arrangement and adaptation were hardly distinguished. On the contrary, all three were embedded in the process of preparing performances.

Sallis, Music Sketches, 37.

${ }^{14}$ Frederick Neumann, «The Use of Baroque Treatises on Musical Performance», Music \& Letters 48, n. 4 (1967): 315-324.

${ }^{15}$ Neumann, «The Use of Baroque Treatises...», 317.

${ }^{16}$ En el caso de Pisendel encontramos significativas diferencias entre lo que los tratados y las fuentes impresas cercanas nos dicen y las prácticas del propio Pisendel. Estas diferencias han sido analizadas en dos artículos del autor: Javier Lupiáñez, «Personal Manuscripts as Sources for Ornamentation: the Embellishments of Pisendel in Schrank II, with a Special Focus on Vivaldi», Early Music Performer 46 (2020): 9-13; y Javier Lupiáñez y Fabrizio Ammetto, «Las anotaciones de Pisendel en el Concierto para dos violines RV 507 de Vivaldi: Una ventana abierta a la improvisación en la obra del "Cura Rojo"», en Musical Improvisation in the Baroque Era (Turnhout: Brepols, 2014), 43-62.

${ }^{17}$ Sallis, Music Sketches, 26.
} 
imaginar otro artista que nos haya legado una cantidad semejante de fuentes que documenten su actividad en todos estos campos. Los manuscritos de sus propias composiciones son una muestra de cómo los papeles de compositor e intérprete se diluyen en muchas ocasiones pero también toman posiciones completamente separadas en otras y arrojan luz sobre interesantes cuestiones interpretativas.

\section{I.2. La colección personal de Johann Georg Pisendel, el Schrank II}

Pisendel entró a formar parte de la orquesta de la corte de Dresde en 1712, ${ }^{18}$ y tomó el papel de concertino en 1728, aunque no fue oficialmente reconocido como tal hasta $1730 .{ }^{19}$ A su muerte, en 1755, Pisendel dejó un inmenso archivo del que han sobrevivido aproximadamente 1800 partituras. ${ }^{20}$ Sabemos que desgraciadamente gran parte de la colección original se perdió entre las llamas del sitio de Dresde ${ }^{21}$ y los bombardeos de las tropas prusianas en $1760 .{ }^{22}$ Las partituras restantes fueron trasladadas a los sótanos de la Katholischen Hofkirche de Dresde donde fueron almacenadas en el armario número «dos» (Schrank II en alemán), ordenadas en un meticuloso orden alfabético.

Tras cien años de completo olvido, el compositor, director y violonchelista Julius Rietz (1812-1877), que por aquel entonces ostentaba el cargo de director musical de la ciudad de Dresde redescubrió el archivo. ${ }^{23}$ Tras el descubrimiento, Moritz Fürstenau (1824-1889), ${ }^{24}$ encargado de la Königliche Privatmusiksammlung y flautista de la Hofkapelle fue quien tomó la tarea de asignar nuevos números de archivo a las partituras e incluirlas en la colección real (Königliche Privatmusiksammlung).

Entre la gran variedad y heterogeneidad de la colección de Pisendel en el Schrank II de la Sächsische Landesbibliothek — Staats- und Universitätsbibliothek Dresden (SLUB) encontramos documentos musicales de naturaleza personal. Se trata de partituras que contienen todo tipo de

${ }^{18}$ Kai Köpp, Johann Georg Pisendel (1687-1755) und die Anfange der neuzeitlichen Orchesterleitung (Tutzing: Hans Schneider, 2005), 79.

${ }^{19}$ Köpp, Johann Georg Pisendel..., 236.

${ }^{20}$ «Schrank II», en Hofmusik in Dresden, acceso el 26 de junio de 2020, https://hofmusik.slub-dresden.de/ themen/schrank-zwei/.

${ }^{21}$ El sitio de Dresde tuvo lugar en julio de 1760 durante la Tercera Guerra de Silesia (parte de la Guerra de los Siete Años) cuando una fuerza prusiana encabezada por Federico el Grande sitió sin éxito la ciudad de Dresde.

${ }^{22}$ Steven Zohn, «Das instrumental Repertoire der dresdner Hofkapelle in den ersten beiden Dritteln des 18. Jahrhunderts: Überlieferung und Notisten Sächsische Landesbibliothek - Staats- und Universitätsbibliothek, Dresden, 23-25 june 2010», Eighteenth Century Music 8, n. ${ }^{\circ} 1$ (2011): 168-170.

${ }^{23}$ Albert Mell y Matthias Wiegandt, «Rietz, Julius», en Grove Music Online. Oxford University Press, acceso el 25 de junio de 2020, http://www.oxfordmusiconline.com/subscriber/article/grove/music/23451.

${ }^{24}$ Gaynor G. Jones, «Fürstenau family», en Grove Music Online. Oxford University Press, acceso el 25 de junio de 2020, http://www.oxfordmusiconline.com/subscriber/article/grove/music/10402pg3. 
anotaciones del propio Pisendel: ideas para la ornamentación, ${ }^{25}$ digitaciones, anotaciones para liderar la orquesta, arreglos propios de otros compositores o bocetos e ideas para sus propias composiciones, etc.

\section{Pisendel SE INTERPRETA A Sí Mismo}

El catálogo compositivo de Johann Georg Pisendel es muy pequeño y ni siquiera las nuevas adiciones que se han hecho en los últimos años cambian ese hecho. ${ }^{26}$ La lista de piezas atribuidas a Pisendel no llega a la treintena, lo cual es muy poco si tenemos en cuenta que estuvo al menos veintiocho años encargado de la música instrumental de la corte de Dresde, ${ }^{27}$ un cargo que requería de Pisendel «conseguir y arreglar música propia y de otros compositores $»^{28}$.

Es muy significativo que un gran porcentaje de los manuscritos que contienen anotaciones interpretativas en la colección del Schrank II sean precisamente composiciones del propio Pisendel.

En el Schrank II, un total de $156^{29}$ manuscritos contienen algún tipo de anotación para la ornamentación, de estos, 36 contienen obras de Vivaldi, 23 manuscritos contienen obras de compositores anónimos, 24 contienen obras de Graun, 16 obras de Fasch y 22 manuscritos contienen obras de Pisendel. Los siguientes compositores con manuscritos que contienen anotaciones están ya lejos de estas cifras: Telemann con 5 manuscritos y Handel, Quantz y Geminiani con 3 manuscritos cada uno son los siguientes de la lista.

Hay que decir que aunque parece obvio que se trata de un archivo de gran riqueza en cuanto a fuentes para la ornamentación, se han realizado muy pocos estudios sobre el lenguaje interpretativo de Pisendel ligado a la ornamentación o dedicados al estudio de sus anotaciones. El temprano estudio llevado a cabo en 1909 por Schering ${ }^{30}$ donde se analizan algunas de las anotaciones de Pisendel, especialmente las dedicadas al concierto de Vivaldi RV 202, no tuvo continuación hasta los trabajos de

${ }^{25}$ Lupiáñez, «Personal Manuscripts ...., 9-13.

${ }^{26}$ Manfred Fechner, «Works in Progress. Pisendels Concerti», en Johann Georg Pisendel : Studien zu Leben und Werk ; Bericht über das internationale Symposium vom 23. bis 25. Mai 2005 in Dresden, ed. por Ortrun Landmann y HansGünter Ottenberg (Hildesheim: Georg Olms Verlag AG, 2010), 144.

${ }^{27}$ Köpp, Johann Georg Pisendel..., 383.

${ }^{28}$ [...] mit seinen eigenen und anderen compositionen die Music zu bestellen und zu dirigiren.

Köpp, Johann Georg Pisendel..., 383.

${ }^{29}$ La siguiente enumeración es de elaboración propia y muestra mis conclusiones a la hora de la elaboración de este artículo, no se descartan nuevos hallazgos en el futuro.

30 Arnold Schering, "Zur instrumentalen Verzierungskunst im 18. Jahrhundert'», en Sammelbande der Internationalen Musikgesellschaft Siebenter Jahrgang 1905-1906, ed. por Max Seiffert (Leipzig: Breitkopf \& Härtel, 1906), 365-385. 
Fechner ${ }^{31}$ casi setenta años después. Poco o nada se ha escrito sobre los ornamentos y las anotaciones de Pisendel en el repertorio escrito por él mismo. Y eso que el porcentaje de manuscritos con anotaciones en el Schrank II es en el caso de Pisendel el mayor de todos, con muchísima diferencia.

Tabla 1. Relación de los compositores que tienen mayor número de manuscritos con anotaciones de Pisendel para la ornamentación y porcentaje de manuscritos con anotaciones con respecto al total de manuscritos por autor en la colección de Pisendel

\begin{tabular}{|l|l|l|l|}
\hline Compositor & $\begin{array}{l}\text { Manuscritos totales en el } \\
\text { Schrank II }\end{array}$ & $\begin{array}{l}\text { Manuscritos con } \\
\text { anotaciones }\end{array}$ & $\begin{array}{l}\text { Porcentaje de mss. } \\
\text { anotaciones }\end{array}$ \\
\hline Vivaldi & 168 & 36 & $21,4 \%$ \\
\hline Graun & 165 & 24 & $14,5 \%$ \\
\hline Anónimos & 190 & 23 & $12 \%$ \\
\hline Fasch & 183 & 16 & $8,7 \%$ \\
\hline Pisendel & $47^{\text {a }}$ & 22 & $46,8 \%$ \\
\hline
\end{tabular}

${ }^{a}$ Este número se basa en un listado realizado por el autor. Aún falta por elaborar un catálogo exhaustivo de la producción de Pisendel. Se incluyen en el recuento algunos movimientos sueltos insertados en otras piezas aunque se trata, casi sin duda, de un catálogo todavía incompleto.

Se elabora a continuación una lista detallada de elaboración propia con las obras y manuscritos donde Pisendel se ornamenta a sí mismo:

Tabla 2. Lista con las obras de Pisendel que contienen anotaciones para la ornamentación

\section{Obra}

\begin{tabular}{|l|l|l|}
\hline Fuente (Formato) & Fecha & Copista $^{\text {b }}$ \\
\hline Anotaciones & \\
\hline
\end{tabular}

${ }^{\mathrm{b}}$ Gracias al proyecto de digitalización y estudio de los manuscritos contenidos en el Schrank II llevado a cabo por la SLUB de Dresde es posible encontrar un catálogo detallado de los copistas involucrados en cada manuscrito. Este catálogo está además conectado con el RISM, que ofrece información sobre los copistas en Dresde siempre que sea posible. Es posible consultar el catálogo de copistas en el siguiente enlace: https://hofmusik.slub-dresden.de/en/ catalogues/writers-catalogue/.

${ }^{31}$ Manfred Fechner, «Improvisationsskizzen und ausnotierte Diminutionen von Johann Georg Pisendel, dargestellt an in Dresden handschriflich überlieferten Konzerten von Johann Friedrich Fasch und Johann Gottlieb Graun», en Zu Fragen der Improvisation in der Instrumentalmusik der ersten Hälfe des 18. Jabrbunderts. Konferenzbericht der 7. Wissenschaflichen Arbeitstagung Blankenburg/Harz, 29. Juni bis 1. Juli 1979, ed. por Eitelfriedrich Thom (Blankenburg/ Harz: Die Kultur-und Forschungsstätte, 1980), 35-55. 


\section{Concierto para violín en fa mayor}

\begin{tabular}{|c|c|c|c|}
\hline & Mus.2421-O-13 (Partitura) & $1720-1740$ & Pisendel \\
\hline & \multicolumn{3}{|c|}{ Anotaciones para la ornamentación en todos los movimientos. } \\
\hline & Mus.2421-O-13a (Partes) & $1720-1735$ & Pisendel $^{\mathrm{c}}$ y Copista $\mathrm{k}$ \\
\hline & \multicolumn{3}{|c|}{ Anotaciones para la ornamentación en todos los movimientos. } \\
\hline & Mus.2421-O-13b (Partes) & $1720-1745$ & Grundig \\
\hline & \multicolumn{3}{|c|}{ Anotaciones para la ornamentación en todos los movimientos. } \\
\hline \multicolumn{4}{|c|}{ Concierto para violín en la menor (un movimiento) } \\
\hline & Mus.2421-O-14 (Partitura) & ca. 1717 & Pisendel y Vivaldi \\
\hline & \multicolumn{3}{|c|}{$\begin{array}{l}\text { Correcciones de Vivaldi y varias anotaciones de Pisendel para la ornamentación. } \\
\text { En un folio aparte encontramos bocetos y anotaciones por identificar. }\end{array}$} \\
\hline \multicolumn{4}{|c|}{ Concierto para violín en si bemol mayor } \\
\hline & Mus.2421-O-12d (Partitura) & $1720-1745$ & Pisendel \\
\hline & \multicolumn{3}{|c|}{ Anotaciones para la ornamentación en el último movimiento. } \\
\hline \multicolumn{4}{|c|}{ Concierto para violín en mi bemol mayor JunP I.3 } \\
\hline & Mus.2421-O-7a $\mathrm{a}^{\mathrm{e}}$ (Partes) & $1735-1745$ & Pisendel $^{\mathrm{f}}$ y Copista $\mathrm{M}$ \\
\hline & \multicolumn{3}{|c|}{ Anotaciones para la ornamentación en todos los movimientos. } \\
\hline & Mus.2421-O-7 (Partitura) & $1720-1735$ & Pisendel \\
\hline & \multicolumn{3}{|c|}{$\begin{array}{l}\text { Correcciones de tipo compositivo. Algunas anotaciones para el segundo y } \\
\text { tercer movimiento escritas en el folio 3r. (Al final del primer movimiento) y } \\
\text { gran cantidad anotaciones en la última página (fol. } 5 \mathrm{v} \text {.) para la ornamentación } \\
\text { del primer movimiento. }\end{array}$} \\
\hline \multicolumn{4}{|c|}{ Concierto para violín y orquesta en mi menor } \\
\hline & Mus.2421-O-15 (Partitura) ${ }^{\mathrm{g}}$ & $1720-1735$ & Pisendel \\
\hline & \multicolumn{3}{|c|}{$\begin{array}{l}\text { Muchas anotaciones para la ornamentación en todos los movimientos junto } \\
\text { con correcciones de tipo compositivo. }\end{array}$} \\
\hline
\end{tabular}

\footnotetext{
c Anotaciones en la parte copiada por Pisendel.

${ }^{\mathrm{d}}$ Existe otra fuente sin anotaciones para la ornamentación: Mus.2421-O-12a.

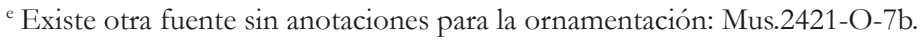

${ }^{\mathrm{f}}$ Anotaciones en la parte copiada por Pisendel.

g Otras fuentes sin anotaciones: Mus.2-O-1,41a y Mus.2-O-1,41b.
} 


\section{Concierto para violín en re mayor JunP I.7.b, JunP II.3}

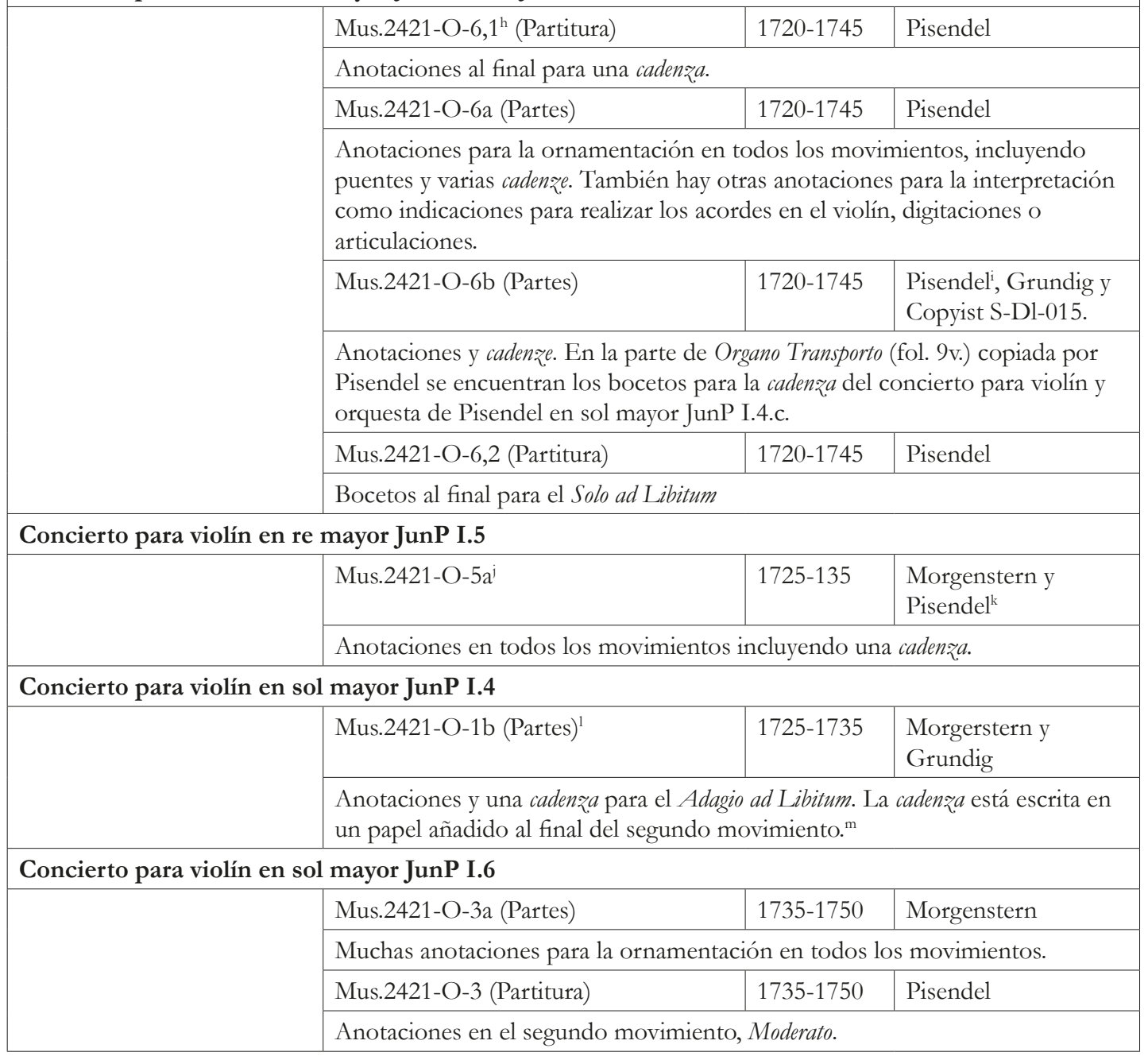

\footnotetext{
${ }^{\text {h }}$ Otras fuentes sin anotaciones: D-Bsa SA 2873.

${ }^{i}$ Anotaciones en la parte copiada por Pisendel.

¡ Otras fuentes sin anotaciones: Partitura en Mus.2421-O-5.

${ }^{k}$ Anotaciones en la parte copiada por Pisendel.

${ }^{1}$ Otras fuentes: Mus.2421-O-1, Mus.2421-O-1a y D-Bsa SA 2799(11).

${ }^{\mathrm{m}}$ Los bocetos para esta cadenza se encuentran en otro manuscrito, el Mus.2421-O-6b, fol. 9v.
} 


\section{Concierto para violín en sol menor JunP I.1}

\begin{tabular}{|c|c|c|c|}
\hline & Mus.2421-O-11 (Partitura) & $1720-1745$ & Pisendel \\
\hline & \multicolumn{3}{|c|}{ Anotaciones para la ornamentación en todos los movimientos. } \\
\hline & Mus.2421-O-10 (Partes) & $1725-1735$ & Morgenstern \\
\hline & \multicolumn{3}{|c|}{ Anotaciones para la ornamentación en todos los movimientos } \\
\hline \multicolumn{4}{|c|}{ Sonata para violín en do menor } \\
\hline & D-Dl Mus.2201-R-11 $\mathrm{a}^{\mathrm{n}}$ & $1720-1740$ & Pisendel \\
\hline & \multicolumn{3}{|c|}{ Correcciones compositivas y anotaciones para la ornamentación. } \\
\hline \multicolumn{4}{|c|}{ Sonata para violín en mi mayor } \\
\hline & Mus.2421-R-18 & $1716-1720$ & Pisendel \\
\hline & \multicolumn{3}{|c|}{ Correcciones compositivas y anotaciones para la ornamentación. } \\
\hline \multicolumn{4}{|c|}{ Sonata para violín en mi menor JunP IV.1 } \\
\hline & Mus.2421-R-5 & $1720-1755$ & Pisendel \\
\hline & \multicolumn{3}{|c|}{ Correcciones compositivas y anotaciones para la ornamentación. } \\
\hline \multicolumn{4}{|c|}{ Sonata para violín en sol menor } \\
\hline & Mus.2421-R-15 & $1720-1755$ & Pisendel \\
\hline & \multicolumn{3}{|c|}{$\begin{array}{l}\text { Correcciones compositivas y anotaciones para la ornamentación. En un folio } \\
\text { a parte encontramos los bocetos para la sonata de Pisendel en mi mayor } \\
\text { contenida en el Mus.2421-R-18. }\end{array}$} \\
\hline
\end{tabular}

${ }^{\mathrm{n}}$ Otras fuentes: D-D1 Mus.2201-R-11 y Mus.2-R-8.34.

${ }^{\circ}$ Otras fuentes: Mus.2421-R-6 y Mus.2421-R-1.

Casi todas las obras que Pisendel escribió para violín solista están ornamentadas por él mismo, solamente nos faltan en la lista su sonata para violín y continuo en re mayor, ${ }^{32}$ la sonata en mi bemol mayor, ${ }^{33}$ la sonata para violín solo, ${ }^{34}$ el concierto para violín y orquesta JunP II. ${ }^{35}$ y el concierto para violín y orquesta en mi bemol mayor. ${ }^{36}$

Cuando vemos todas estas anotaciones sobre la partitura podemos afirmar que nos encontramos ante lo que podríamos denominar los «manuscritos de trabajo» ${ }^{37}$ que Pisendel elaboró

${ }^{32}$ Mus.2421-R-9.

${ }^{33}$ Mus.2421-R-12.

${ }^{34}$ Mus.2421-R-2.

${ }^{35}$ Mus.2421-O-2.

${ }^{36}$ Mus.2421-R-9.

${ }^{37}$ Usamos este término genérico ya que como bien se apunta en Sallis, Music Sketches..., 240, la terminología en este sentido puede ser problemática cuando define el término boceto como: «DRAFT: A preliminary version of a piece of writing or document. The plethora of terms that have been invented in various languages to seize the different 
para sí mismo y que le servían como preparación de sus propias interpretaciones. Resulta evidente por su tipología y función que todas estas anotaciones están destinadas a ser solamente interpretadas por el mismo Pisendel. Razón por la cual realizar una lectura o una transcripción fidedigna de las mismas es tarea casi imposible. ${ }^{38}$

De todos modos el estudio de estos manuscritos de trabajo revelan interesantes usos de la ornamentación y del papel de Pisendel como intérprete y como compositor.

\section{II.1. Ejemplos significativos}

La colección de Pisendel nos ofrece un amplio abanico de fuentes; de algunas obras solo nos han llegado los manuscritos de trabajo de Pisendel, de otras han llegado hasta nosotros únicamente los manuscritos copiados a limpio. Afortunadamente encontramos algunas piezas contenidas en varias fuentes, cada una de ellas nos ofrece diferente información sobre la pieza, desde los manuscritos de trabajo, repletos de enmiendas compositivas e interpretativas hasta la copia limpia, destinada a su difusión o archivo. Para los siguientes ejemplos se han seleccionado piezas que se encuentran contenidas tanto en manuscritos de trabajo como en versiones limpias, lo que nos permite la comparación entre las fuentes. Además, de entre la gran cantidad de interesantes ejemplos de ornamentación que nos ha dejado Pisendel, tomaremos algunos de los más elaborados. Estos ejemplos nos muestran un uso muy interesante de la ornamentación. Estos usos incluyen la ornamentación en movimientos rápidos, en ocasiones desde la primera nota de la parte solista, un rico estilo en los movimientos lentos y la inclusión de cadenže.

\section{II.1.1. Ornamentando desde la primera nota}

Pocas variaciones extempore están permitidas en el Allegro, ya que generalmente éste se forma con melodías y pasajes de un tipo que dejan poco margen a la mejora $[. .$.$] al hacer esto [agregando$ variaciones al Allegro] los artistas a menudo estropean más de lo que mejoran. Pero si aún desean hacer algunas variaciones, no deben hacerlo antes de la repetición. ${ }^{39}$

stages of the creative process between sketches and the fair copy is problematic».

${ }^{38}$ Nicholas Scott Lockey, «Second Thoughts, Embellishments and an Orphaned Fragment: Vivaldi's and Pisendel's Contributions to the Dresden Score of RV 340», Studi vivaldiani 10 (2010): 125-142.

${ }^{39}$ Few extempore variations are allowed in the Allegro, since it is usually with melodies and passages of a kind that leave little room for improvement [...] by doing this [adding variations to the Allegro] performers often spoil more than they improve. But if you still want to make some variations, you must not do so before the repetition.

Johann Joachim Quantz, On playing the Flute. Trad. por Edward R. Reilly (Londres: Faber and Faber, 1985), 134. 
Contrariamente a esta cita de Quant ${ }^{40}$ encontramos ornamentaciones sobre movimientos rápidos en toda la colección de Pisendel en el Schrank II, al menos unos 68 manuscritos ${ }^{41}$ contienen algún tipo ornamentación en los movimientos rápidos. Especialmente las composiciones de Pisendel presentan una gran cantidad de ideas para ornamentar los movimientos rápidos de sus conciertos.

En ocasiones estas ideas comienzan ya desde la primera nota en la intervención del violín solista. Es lo que ocurre en el concierto para violín en mi bemol mayor JunP I.3 (Contenido en tres fuentes diferentes: Mus.2421-O-7a, Mus.2421-O-7 y Mus.2421-O-7b) y que se muestra en el ejemplo de la imagen $1 .^{42}$ Los ornamentos que encontramos en la partitura de trabajo del Mus.2421-O-7a comienzan desde la primerísima intervención del violín solista, Pisendel no tiene remilgos en comenzar el primer solo del concierto con una versión variada y ornamentada de su propio tema, que además es el tema principal que se repite y articula todo el movimiento.

${ }^{40}$ Quantz publica su Versuch einer Anweisung die Flöte traversiere zu spielen en Berlín en 1752. Aunque esto nos posiciona al final de la vida de Pisendel (que muere en 1755) la importancia de Quantz para entender el contexto interpretativo de Pisendel es fundamental. No solo Quantz y Pisendel disfrutaron de una relación profesional y de amistad sino que los textos de Quantz podrían haber sido influenciados directamente por Pisendel según las investigaciones de Kai Köpp. Kai Köpp, «Das Testament des Dresdner Konzertmeisters Johann Georg Pisendel», en Jabrbuch 1999 der Ständigen Konferenz Mitteldeutsche Barockmusik, ed. por W. Seidel (Eisenach: Wagner, 2000), 60.

${ }^{41}$ Lupiáñez, «Personal Manuscripts...», 8.

${ }^{42}$ Tanto esta transcripción como las que siguen en el artículo han sido realizadas por el autor y buscan clarificar la escritura original. Al tratarse de manuscritos de uso personal esta escritura puede ser en ocasiones confusa y omite mucha información que solo el propio autor sería capaz de descifrar. Se ha intentado mantener la notación original en todo lo posible, de modo que será posible encontrar muchas incongruencias rítmicas en las transcripciones, para hacer la lectura algo más clara se ha modificado el espaciado entre las notas adaptándolas a otras voces. Se han mantenido las uniones entre las notas e intentado reproducir de la forma más fidedigna posible todas las grafías del original, todas las adiciones del editor aparecerán entre corchetes. 
FRANCISCO JAVIER LUPIÁNEZ RUIZ. COMPLETANDO LO INACABADO
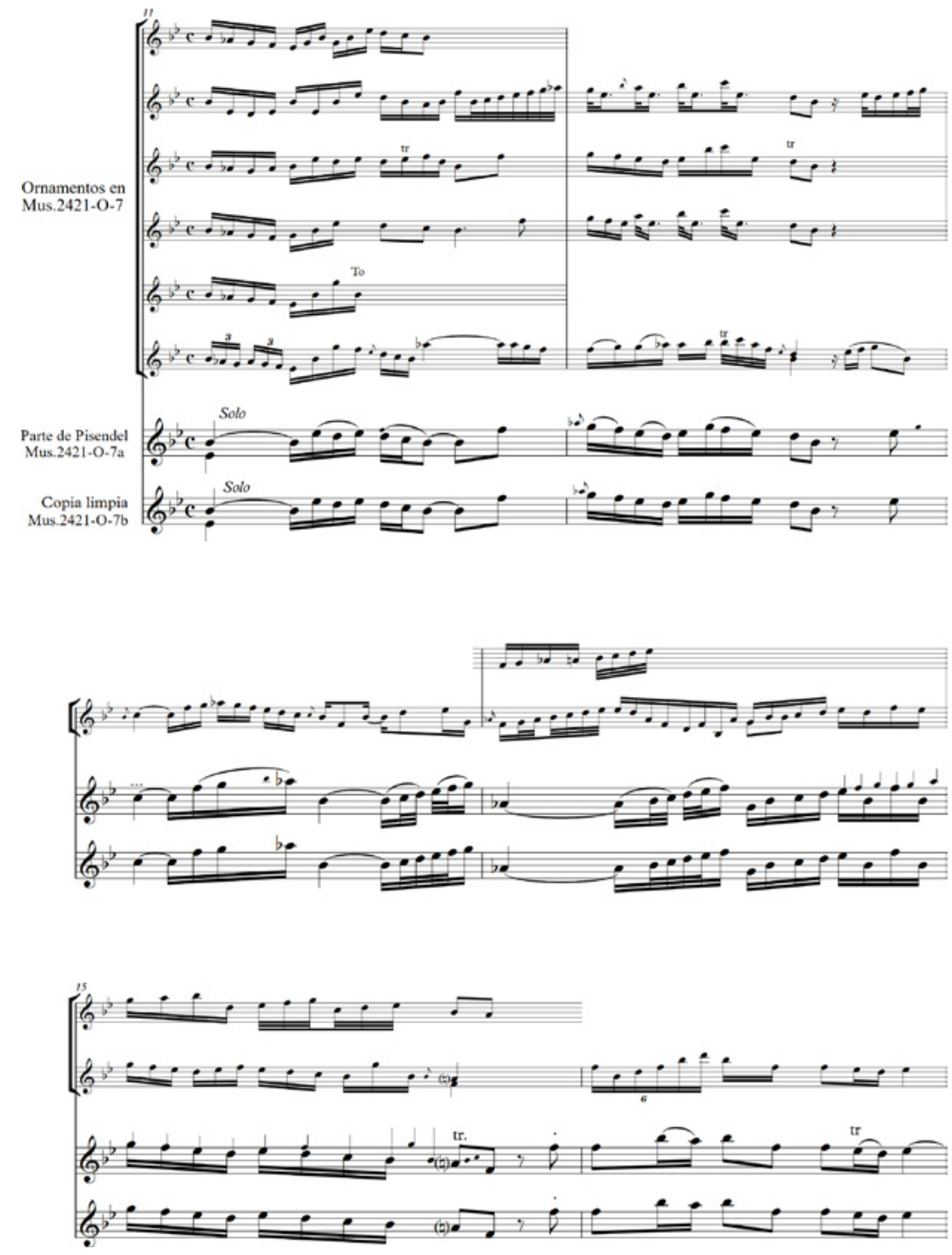

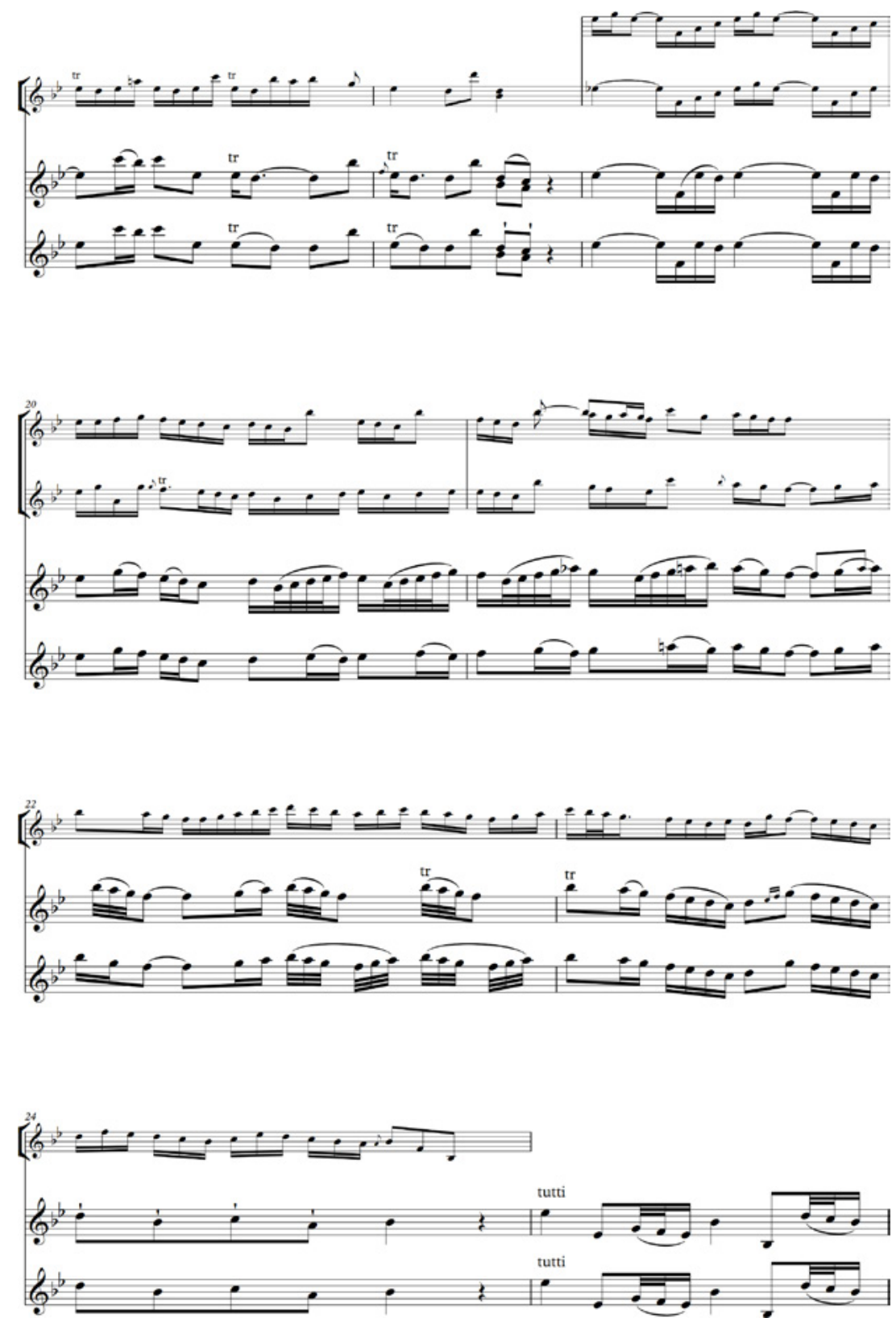

Imagen 1. Pisendel. Concierto para violín en mi bemol mayor JunP I.3.

(Mus.2421-O-7, Mus.2421-O-7a y Mus.2421-O-7b), I, Allegro e sostenuto, cc. 11-25 
Otros conciertos de Pisendel presentan una densidad parecida de ornamentos en los movimientos rápidos. Estas anotaciones suelen encontrarse sobre los manuscritos de trabajo y es muy llamativo que todas estas anotaciones, aunque modifiquen el tema del concierto y sean realmente significativas en términos de cambio del material musical, no se ven reflejadas en el caso de existir una copia a limpio de la obra. Los ejemplos son muy numerosos y no hay espacio aquí para citar siquiera los más interesantes, que son muchos. Veamos como muestra este pasaje del concierto para violín en fa mayor, ${ }^{43}$ que ofrece una variación ostensible de un pasaje de arpegios (variación que solo aparece en la parte que Pisendel usó para la interpretación de la obra).

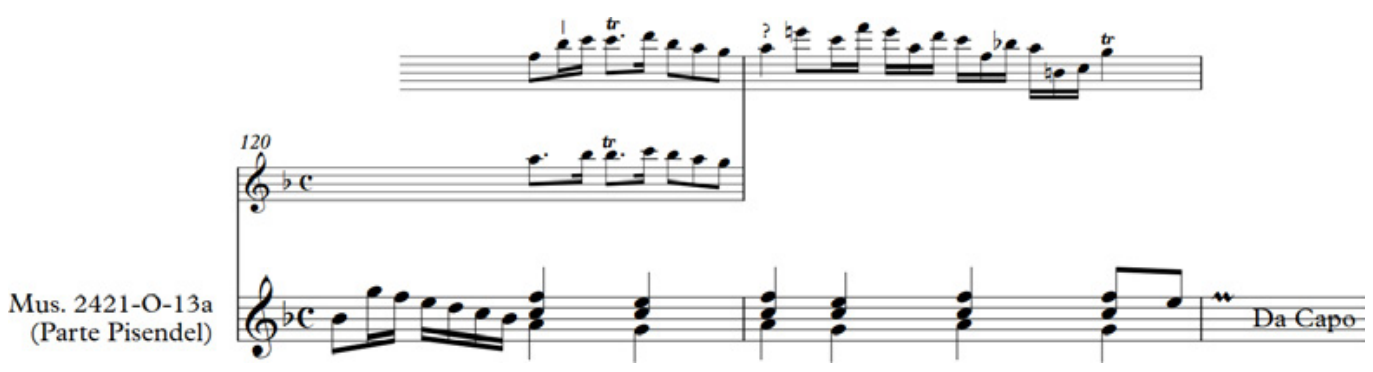

Imagen 2. Pisendel. Concierto para violín y orquesta en fa mayor, Mus.2421-O-13a. I. Allegro, cc. 120-121

Los ejemplos del uso de este tipo de ornamentos en movimientos rápidos en otros compositores son también numerosos. Por citar solo uno, mencionamos aquí el primer solo del concierto para violín RV $302^{44}$ de Vivaldi, contenido en el Mus.2389-O-95, que aparece totalmente ornamentado desde la primera intervención del violín, cambiando, como en el caso del concierto para violín en mi bemol mayor JunP I.3 el motivo que aparecerá como eje temático a lo largo del movimiento.

${ }^{43}$ Existen tres fuentes para este concierto: Mus.2421-O-13 (partitura copiada por Pisendel) Mus.2421-O13a (esta fuente incluye la parte de violín copiada por Pisendel y que seguramente usó en la interpretación debido a la gran cantidad de anotaciones para la interpretación) y Mus.2421-O-13b (otro set de partes copiado por Grundig). Aunque las partes copiadas por Pisendel presentan el mayor número de anotaciones hay que mencionar que incluso la parte copiada por Grundig presenta unas pocas anotaciones, por lo que pudo haber sido usada en algún momento por Pisendel, posiblemente después de haber preparado los ornamentos sobre las otras dos fuentes y ya no le hacían falta tantas anotaciones.

${ }^{44}$ Para una transcripción y análisis de esta fuente véase Lupiáñez, «Personal Manuscripts...», 8. 


\section{II.1.2. Cómo ornamentar los movimientos lentos}

Sirvan unas citas para comenzar esta sección: «Un verdadero músico se distingue por su forma de tocar el adagio» "45; "[De Pisendel] no solo aprendí a tocar el adagio, que tocaba de una manera excepcionalmente conmovedora» ${ }^{46}$; «El adagio lo conmovió aún más: se arrancó la peluca de la cabeza, la tiró al suelo y apenas pudo esperar el final para abrazar a Pisendel con deleite y declararle su admiración» ${ }^{47}$.

Como era normal en aquellos tiempos, los movimientos lentos son un campo especialmente fértil para la ornamentación. Y a tenor de los comentarios de sus contemporáneos, Pisendel era muy bueno a la hora de interpretar este tipo de movimientos. Es interesante observar a través de los manuscritos de sus propias obras las diferencias entre lo que escribió y cómo fue interpretado por él mismo.

Su concierto en mi menor es un caso de estudio perfecto. Encontramos tres fuentes para este concierto: la partitura copiada por Pisendel (Mus.2421-O-15) que contiene gran cantidad de anotaciones en todos los movimientos y dos copias limpias de las partes, una copiada por Pisendel (Mus.2-O-1,41b) y otra por un copista de la corte de Dresde llamado Philipp Troyer (Mus.2-O-1,41a). En el siguiente ejemplo (imagen 3) se transcriben los ornamentos del manuscrito Mus.2421-O-15. Se han organizado las anotaciones que Pisendel realizó sobre el manuscrito de la siguiente manera: La línea 1 indica las ornamentaciones que Pisendel anotó en el pentagrama vacío inferior y la línea 2 indica las ornamentaciones en el pentagrama superior o en pentagramas aparte $(2 \mathrm{a})$.

${ }^{45}$ A true musician may distinguish bimself by the manner in which he plays the Adagio.

Quantz, On playing..., 162.

${ }^{46}$ [...] habe ich nich nur das Adagio, welches er auf eine ausnehmend rübrende Art spielte, vorzutragen gelernet.

Johann Joachim Quantz, «Autobiographischer Abriß», en Historisch-Kritische Beyträge zur Aufnabme der Musik, ed. por Friedrich Wilhelm Marpurg (Berlín: Joh. Jacob Schützens sel. Wittwe, 1754), 211.

${ }^{47}$ Noch mehr würkte das Adagio auf ihn: er riß, während demselben die Perüque von Kopfe, warf sie auf die Erde, und konnte kaum das Ende erwarten, um Pisendeln vor Entzücken zu umarmen, un ihn seiner Hochtung zu versichern.

Esta fue la reacción de Herr Götze, profesor en el Collegium Musicum de Leipzig después de escuchar a Pisendel tocar por primera vez. La anécdota fue recogida en Jahann Adam Hiller, Wöchentliche Nachrichten und Anmerkungen, die Musik betreffend, vol. 1 (Leipzig: Verlag der Zeitungs-Expedition, 1766), 279. 

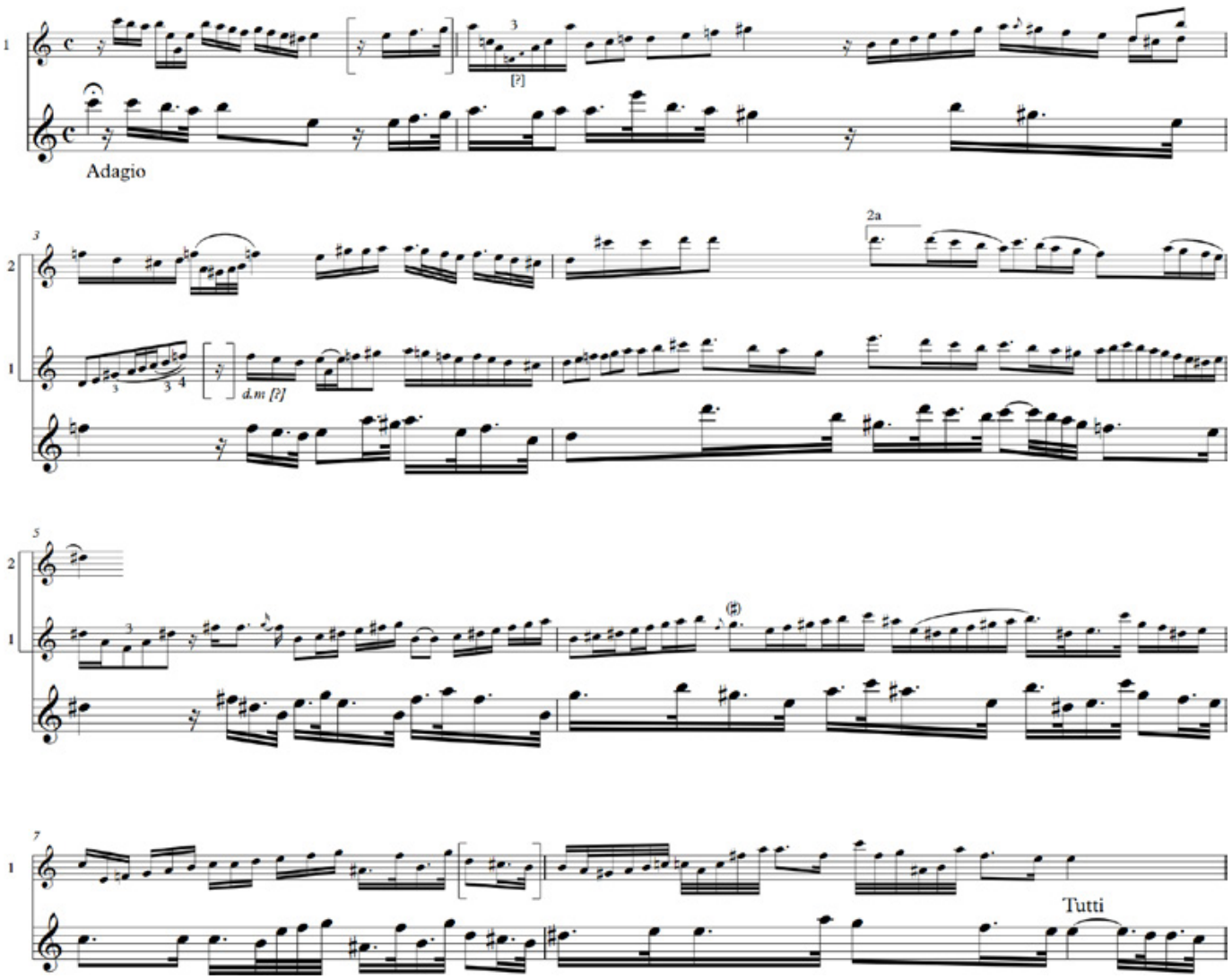

Imagen 3. Pisendel. Concierto para violín y orquesta en mi menor, III. Andante con las disminuciones del Mus.2421-O-15

Pisendel planea una ornamentación para su propia pieza que, a primera vista, poco deja del material original. Pocas notas quedan sin ornamentar (lo cual no quiere decir que no lo fueran) y el ritmo punteado que domina todo el movimiento parece disolverse en las disminuciones.

Otro buen ejemplo que merece ser transcrito es el del concierto para violín en re mayor JunP I.7. Existen cinco fuentes para este concierto (D-Bsa SA 2873, D-Dl Mus.2421-O-6,1, D-Dl Mus.2421-O-6a, D-Dl Mus.2421-O-6b y D-Dl Mus.2421-O-6,2), todas meritorias de un estudio exhaustivo. Sin embargo nos vamos a centrar en el andante que aparece solamente en las Mus.2421-O-6a y en Mus.2421-O-6b; ambas son partes copiadas por Pisendel y ambas presentan una inmensa cantidad de anotaciones. 
La imagen 4 muestra la transcripción del segundo movimiento (Andante) del concierto para violín en re mayor JunP I.7. Mus.2421-O-6a y Mus.2421-O-6b. La transcripción aúna las dos fuentes para que sea más sencillo la comparación y el estudio de las anotaciones. Las líneas se han distribuido de la siguiente manera:

Para el manuscrito Mus.2421-O-6a:

- Mus.2421-O-6a muestra la parte copiada por Pisendel y las anotaciones realizadas directamente sobre la parte.

- La línea 1 muestra las anotaciones realizadas sobre la parte de basso.

- Las líneas 2, 2a, 2b y 2c muestran las anotaciones realizadas al final de la página.

Para el manuscrito Mus.2421-O-6b:

- Mus.2421-O-6b muestra la parte copiada por Pisendel y las anotaciones realizadas directamente sobre ella.

- La línea 3 muestra anotaciones en la parte de basso.

- La línea 3a muestra anotaciones en la parte de violín usando espacio libre al final de la parte.

- Las líneas 3b y 3c muestran las anotaciones hechas en un espacio vacío justo al final del primer movimiento. 

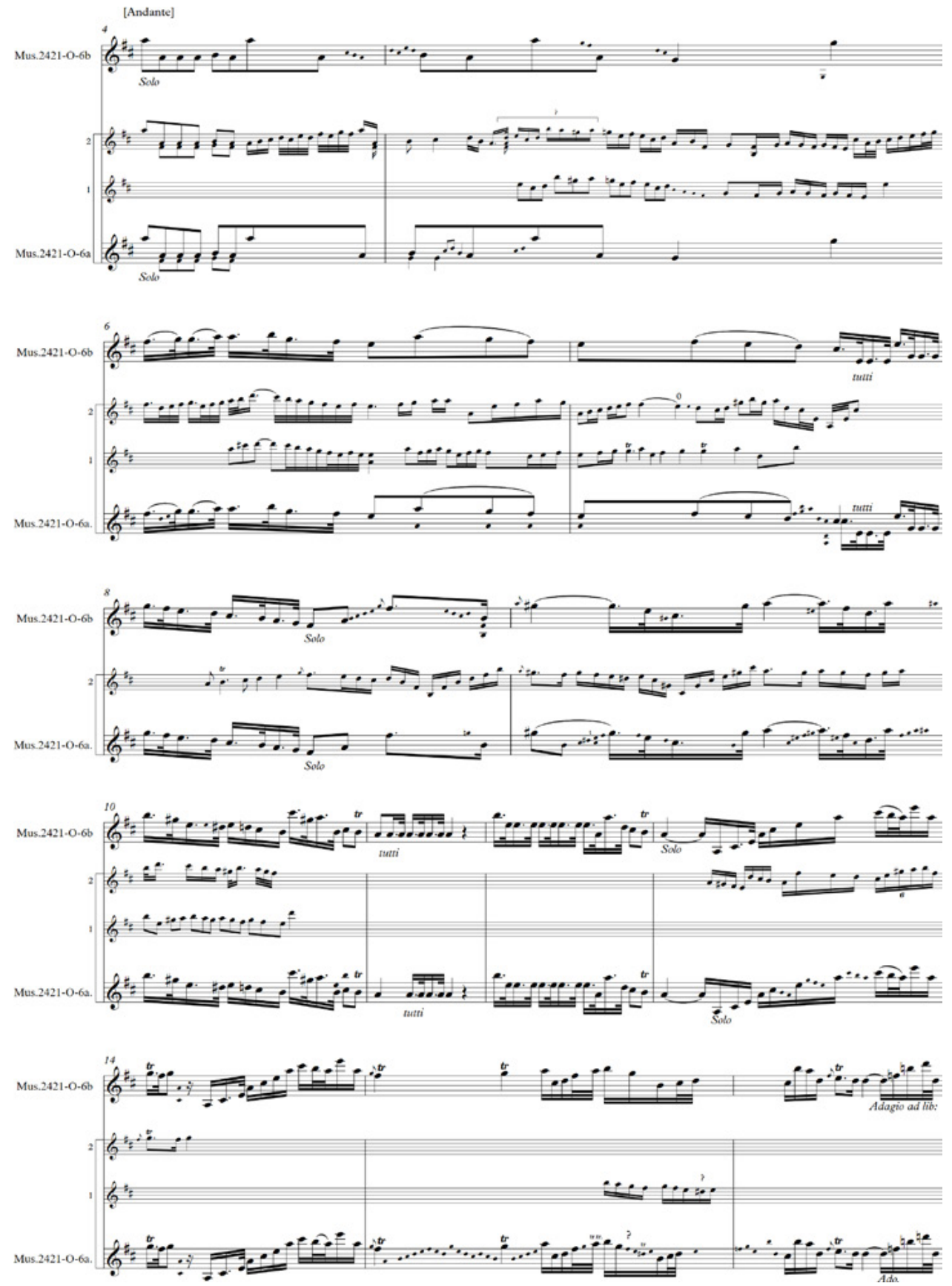


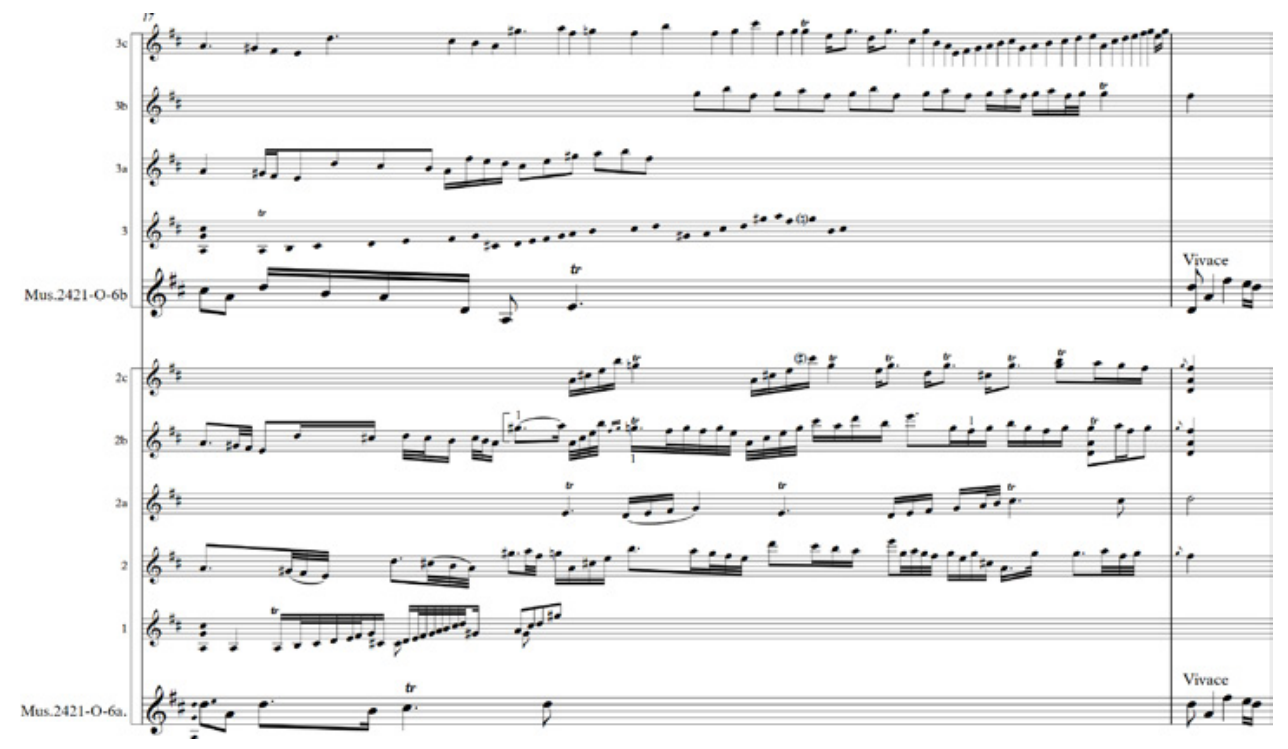

Imagen 4. Pisendel. Concierto para violín en re mayor JunP I.7. Mus.2421-O-6a y Mus.2421-O-6b. II. Andante (Solo)

Existen numerosos ejemplos de estas ricas ornamentaciones en el Schrank II. Otro que merece la pena mencionar es el de las exuberantes disminuciones que Pisendel preparó para el concierto para violín RV 202 de Vivaldi contenido en los manuscritos Mus.2389-O-122 y Mus.2389-O-47a. ${ }^{48}$

Podría parecer, como ocurría con los ornamentos en los movimientos rápidos, que tal cantidad de ornamentos van en contra de los consejos de sus propios contemporáneos: «[Si] desea agregar varias notas [al Adagio] aquí y allá en la melodía, nunca debe hacerlo en exceso, para que la notación principal no se oscurezca y la melodía se vuelva irreconocible» ${ }^{49}$.

Sin embargo, una vez más, hay que tener en cuenta los consejos Neumann ${ }^{50}$ en el uso de estos tratados y observar a quién van dirigidos y qué tipo de significado tienen para un lector moderno. Estos ornamentos que pueden parecernos hoy profusos y abundantes podrían ser comedidos y contenidos para un oyente de la época. El primer biógrafo de Pisendel, Hiller, elogia precisamente las cualidades

${ }^{48}$ Para una transcripción y análisis de esta fuente véase Lupiáñez, «Personal Manuscripts...», 9-13.

49 [if] you want to add several notes [to the Adagio] here and there in the melody, you must never do so in excess, lest the principal note be obscured, and the plain air be made unrecognizable.

Quantz, On playing..., 166-167.

${ }^{50}$ Neumann, «The Use of Baroque Treatises...», 315-324. 
de Pisendel a la hora de ornamentar el Adagio en los siguientes términos: "[Pisendel] fue elogiado especialmente por la interpretación del Adagio, que no estaba sobrecargado con adornos innecesarios $»^{51}$.

\section{II.1.3. Cadenze}

Además de encontrarnos en el Schrank II con cadenze muy elaboradas (también llamadas Capriccio o Fantasia $)^{52}$ Pisendel añade pequeñas o medianas cadenæe. Las encontramos en 39 manuscritos del Schrank II, la mayoría en conciertos de Vivaldi (14 manuscritos) y Pisendel (13 manuscritos).

Este tipo de cadenze parecen haber pasado un poco desapercibidas en la literatura musical de la primera mitad del siglo XVIII. ${ }^{53}$ El capítulo de Quantz sobre ellas es, de lejos, el más completo sobre el tema y nos da una definición general de este tipo de pequeñas cadenze: «Trato aquí ese adorno creado extempore, de acuerdo a la fantasía y el placer del intérprete que se interpreta en una parte concertante al final de una pieza en la penúltima nota del bajo» ${ }^{54}$.

Aunque esta definición se adapta a la mayoría de los casos que encontramos en el Schrank II, Pisendel usará estas cadenže en un modo mucho más amplio: empleándolas como conexión con el siguiente movimiento -como vimos en el concierto para violín en re mayor JunP I.7 (imagen 4)- o incluso insertando varias cadenze en un mismo movimiento, como es el caso del Largo del concierto para violín RV 228 de Vivaldi (Mus.2389-O-58b). ${ }^{55}$

Nos detendremos sobre el hecho de que Quantz indica que este tipo de cadenze son improvisadas. El término extempore no solo es usado por Quantz en la definición que encontramos al principio del capítulo sino que se repetirá a lo largo de todo el texto.

Pisendel se anota sus cadenze (o al menos algunas de ellas). Desde pequeñas cadenze a cadenże algo más elaboradas. Incluso encontramos pruebas del trabajo que le tomó componer algunas de ellas, es el caso del Adagio ad Libitum que encontramos al final del primer movimiento de concierto para violín en sol mayor JunP I.4 contenido en el manuscrito Mus.2421-O-1a (imagen 5) ibm gerühmt.

${ }^{51}$ [...] besonders ward der gründliche, nicht mit überflüßigen Zierrathen überhäufte, männliche Vortrag des Adagio an

Jahann Adam Hiller, Lebensbeschreibungen berübmter Musikgelebrten und Tonkünstler neuerer Zeit (Leipzig: Im Verlage der Dykischen Buchhandlung, 1784), 195.

${ }^{52}$ Para más información sobre cadencias elaboradas en el Schrank II véase Javier Lupiáñez y Fabrizio Ammetto, «A New Vivaldi Cadenza in an Anonymous Violin Concerto», Studi Vivaldiani 17 (2017): 190-202.

${ }^{53}$ Rudolf Rasch, «Improvised cadenzas in the cello sonatas Op. 5 by Francesco Geminiani», en Musical Improvisation in the Baroque Era, ed. por Fulvia Morabito (Turnhout: Brepols, 2019), 195.

${ }^{54}$ I treat here of that extempore embellishment created, according to the fancy and pleasure of the performer, by a concertante part at the close of a piece on the penultimate note of the bass.

Quantz, On playing..., 179.

${ }^{55}$ Para más casos véase Lupiáñez, «Personal Manuscripts...», 3-19. 
y Mus.2421-O-1b (imágenes 6 y 7). En el manuscrito Mus.2421-O-1a Pisendel se anota la cadenza donde buenamente puede, comenzando en un trozo de papel pegado en la segunda página (imagen 6) y en unos pentagramas vacíos al final la primera página del solo (imagen 7). Resulta más o menos sencillo unir estos fragmentos en una transcripción de la cadenza que facilite su lectura (imagen 8).

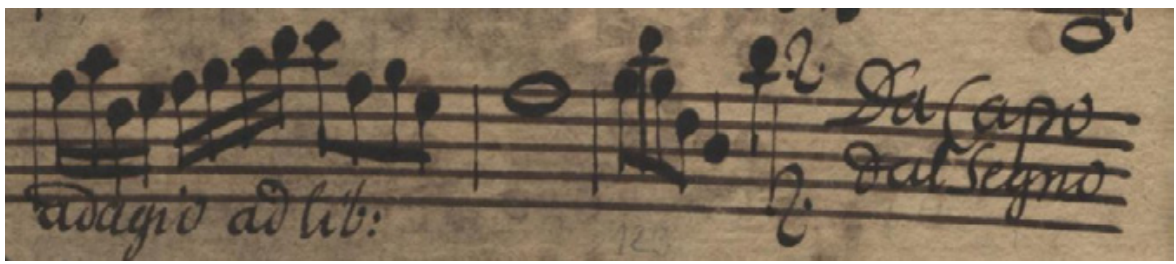

Imagen 5. Pisendel. Concierto para violín en sol mayor JunP I.4, Mus.2421-O-1a. I. Allegro cc. 122-12456

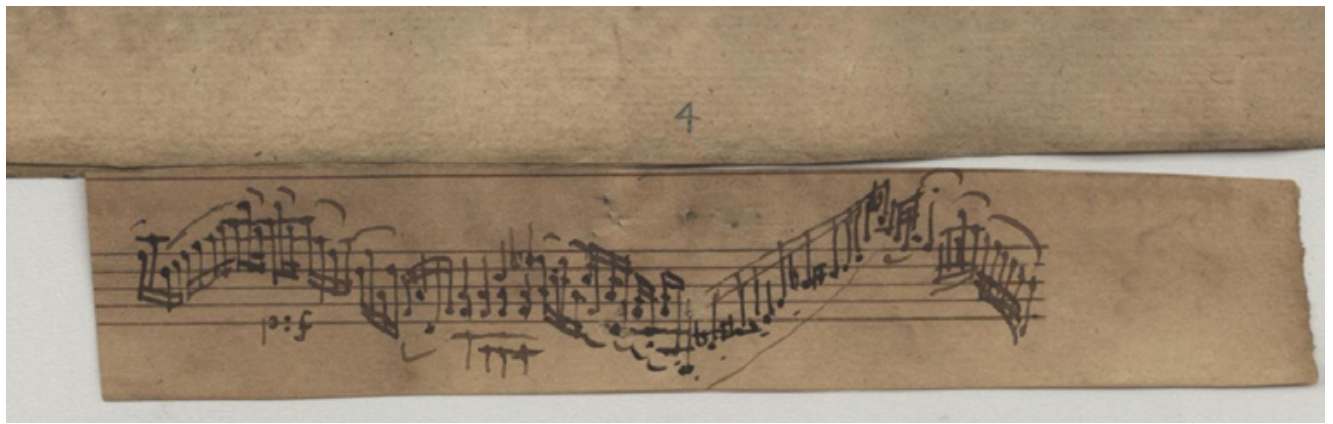

Imagen 6. Cadenza de Pisendel para el Adagio ad Libitum del primer movimento de su concierto para violín en sol mayor JunP I.4. Primera parte de la cadenza anotada en un trozo de papel en la página 8 del manuscrito Mus.2421-O-1b

${ }^{56}$ Agradecemos a la Sächsische Landesbibliothek - Staats- und Universitätsbibliothek Dresden (SLUB) por hacer posible la reproducción de éste y los demás ejemplos de manuscritos originales. Todos ellos pueden consultarse en línea en el proyecto de digitalización del Schrank II llevado a cabo por la SLUB: https://hofmusik.slub-dresden.de/themen/ schrank-zwei/. 


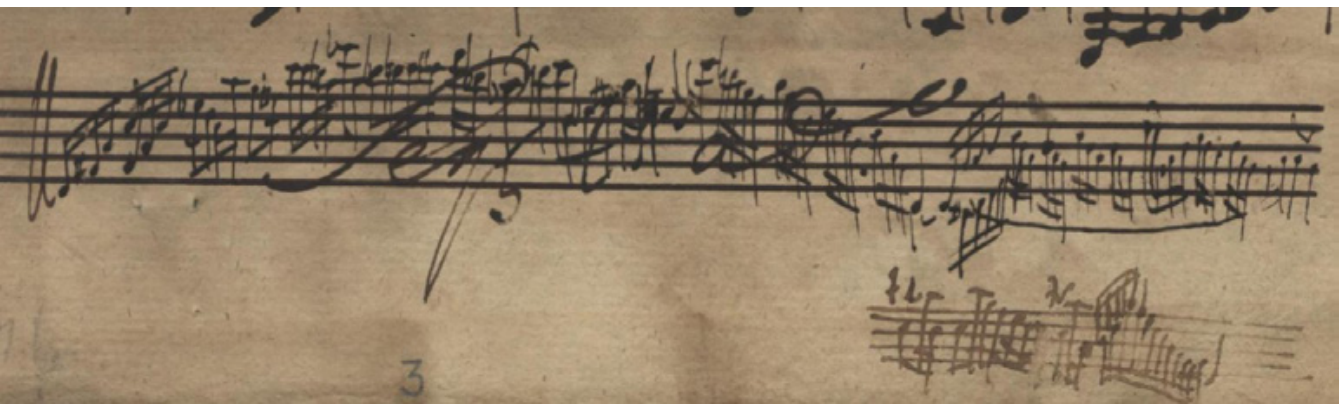

Imagen 7. Cadenza de Pisendel para el Adagio ad Libitum del primer movimento de su concierto para violín en sol mayor JunP I.4. Segunda parte de la cadenža anotada en al final de la página 7 (primera página del solo) del manuscrito Mus.2421-O-1b
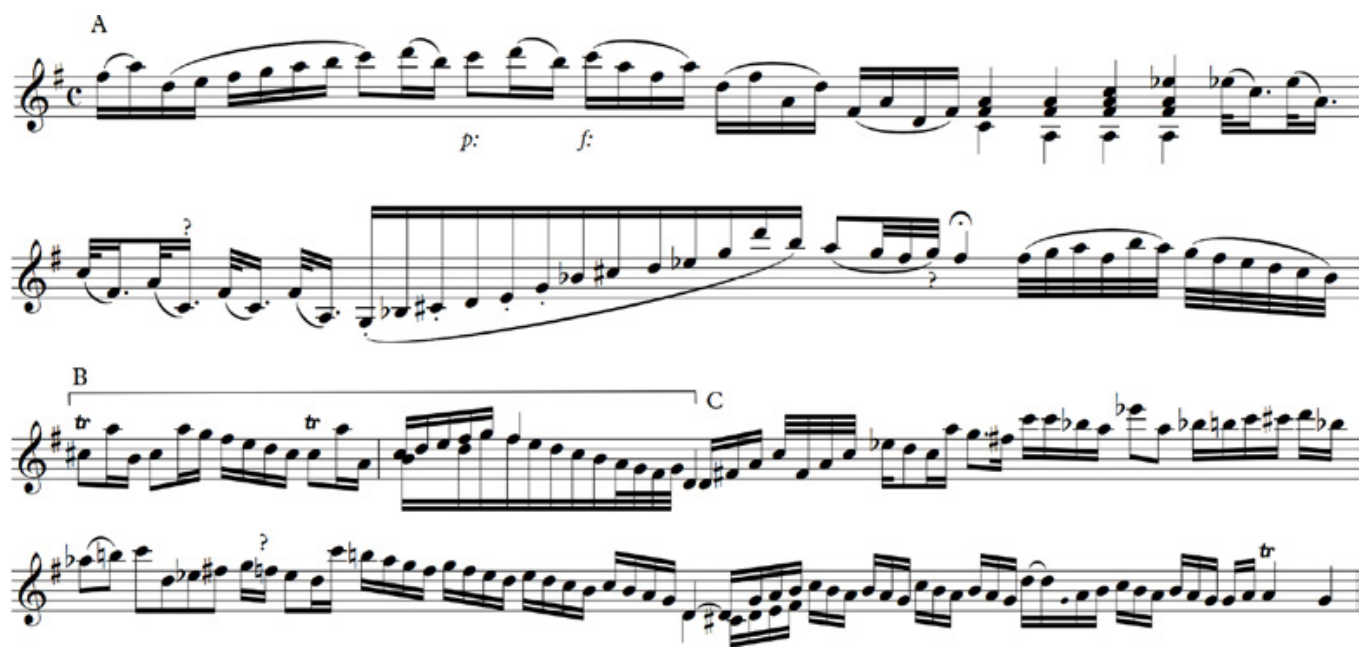

Imagen 8. Pisendel. Concierto para violín en sol mayor JunP I.4. Cadenæa. La sección A del ejemplo se corresponde con la sección contenida en la imagen 6 y las secciones B (anotado aparte) y C con los fragmentos de la cadenza mostrados en la imagen 7

En otro manuscrito, el 2421-O-6b, que contiene el concierto para violín y orquesta de Pisendel en re mayor JunP I.7, justo al final de la parte de Organo Transportato copiada por Pisendel, encontramos lo que parecen ser los bocetos para la composición de esta misma cadenza, unos bocetos que ocupan bastante más que la cadenza en sí (imagen 9). 
FRANCISCO JAVIER LUPIÁÑEZ RUIZ. COMPLETANDO LO INACABADO..

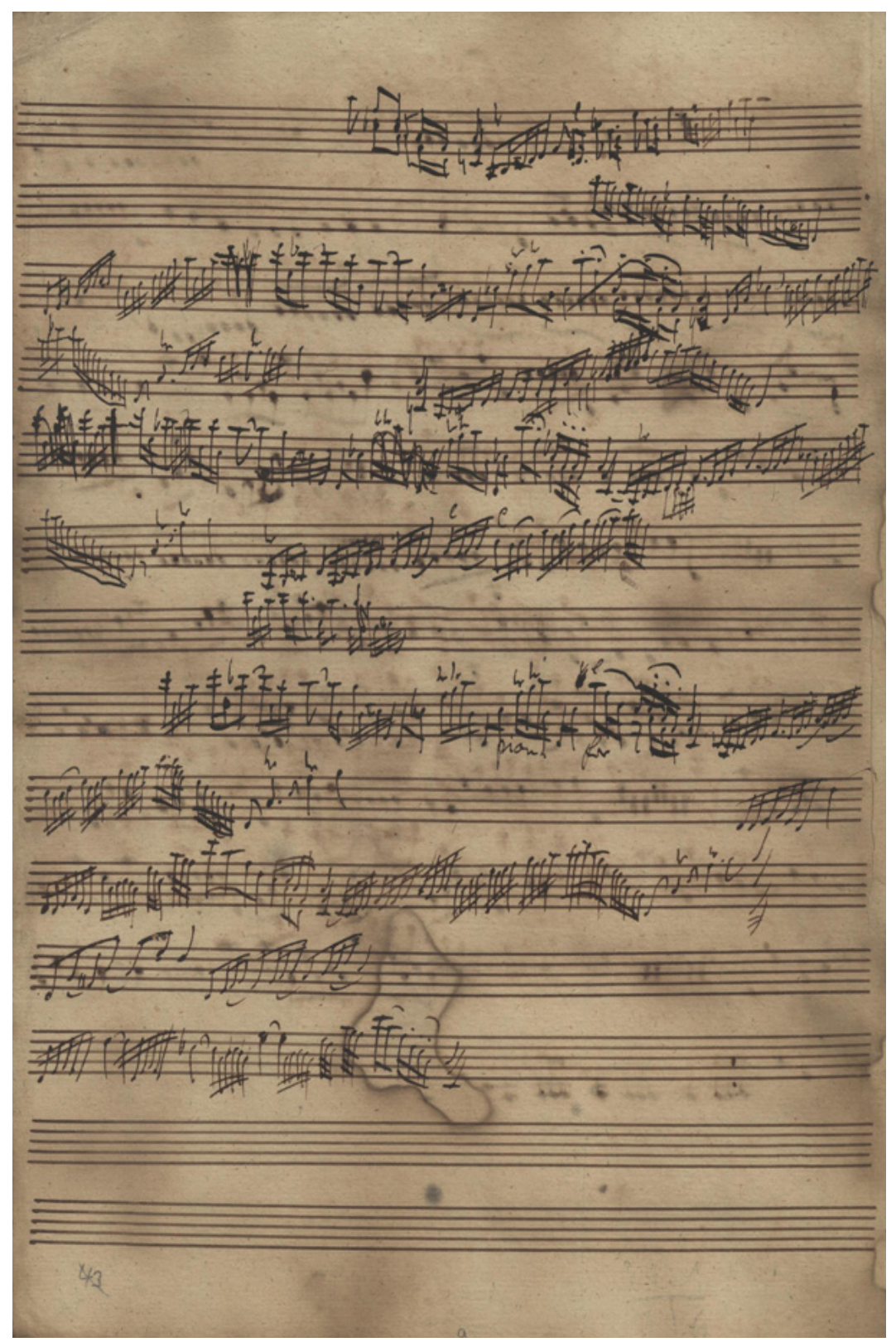

Imagen 9. Bocetos de Pisendel para la cadenza del concierto para violín en sol mayor JunP I.4, Mus.2421-O-6b (fol. 9r.) 
Este tipo de cadenze sobre «pequeños adagios» son comunes en Pisendel y son un muy buen ejemplo de lo que el intérprete recibe en una copia a limpio y lo que se espera de él. En el concierto para violín en re mayor JunP I.7 encontramos otro de estos adagios, el mismo material fue además utilizado en su sonata para violín en re mayor, así que al final tenemos seis fuentes donde Pisendel utiliza este Adagio (D-Bsa SA 2873, D-Dl Mus.2421-O-6,1, D-Dl Mus.2421-O-6a, D-Dl Mus.2421-O-6b y D-Dl Mus.2421-O-6,2 para los conciertos y Mus.2421-R-9 para la sonata)

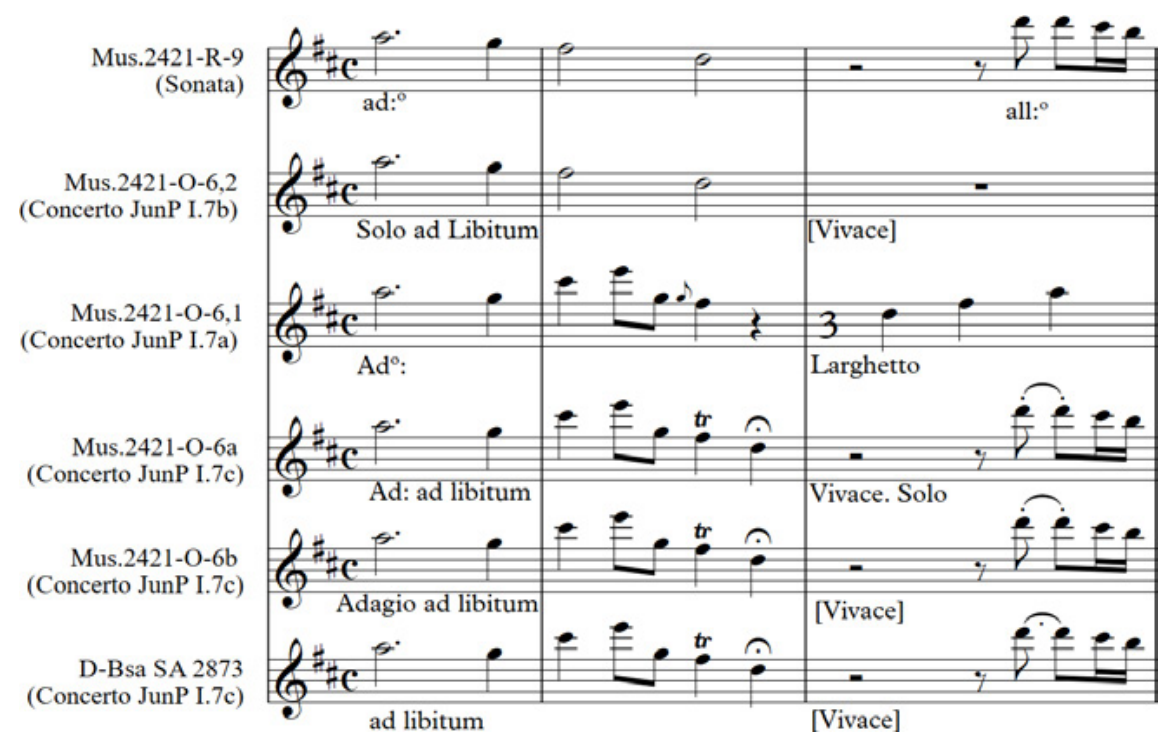

Imagen 10. Diferentes versiones del Adagio en el concierto para violín en re mayor JunP I.7 (primer movimiento cc. 35-37) y la sonata para violín en re mayor (primer movimiento, cc. 4-6)

Este tipo de pasajes son muy interesantes ya que vemos en ellos la estructura melódica dejada por el compositor que debe ser completada (o completamente modificada) por el intérprete, en este caso Pisendel nos dejó sus anotaciones como intérprete para este mismo pasaje en los manuscritos Mus.2421-O-6,1 (imagen 11), Mus.2421-O-6,2 (imagen 12) y Mus.2421-O-6a (imagen 13). 

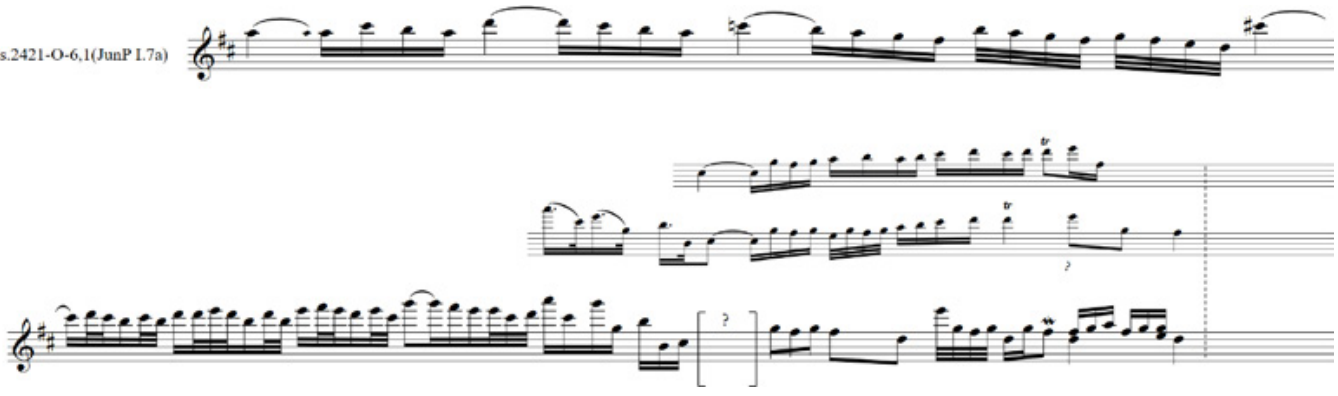

Imagen 11. Ornamentación de Pisendel para el Adagio del concierto para violín en re mayor JunP I.7 en el manuscrito Mus.2421-O-6,1

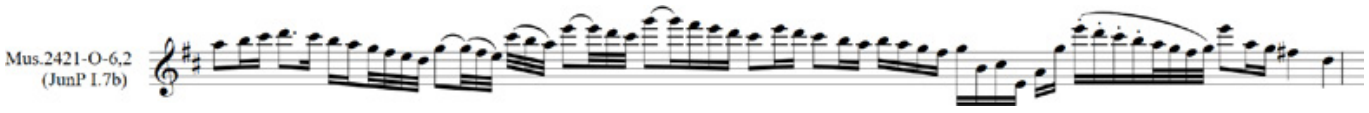

Imagen 12. Ornamentación de Pisendel para el Adagio del concierto para violín en re mayor JunP I.7 en el manuscrito Mus.2421-O-6,2
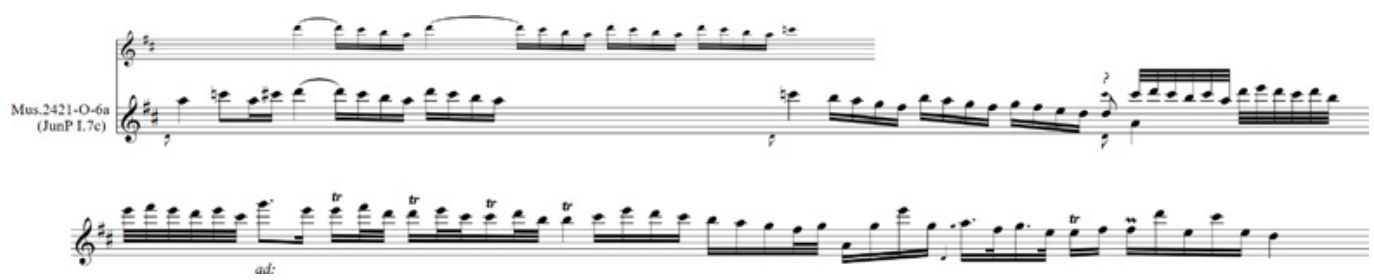

Imagen 13. Ornamentación de Pisendel para el Adagio del concierto para violín en re mayor JunP I.7 en el manuscrito Mus.2421-O-6a

\section{COMPONER LA IMPROVISACIÓN, IMPROVISAR EN LA COMPOSICIÓN}

Quantz insiste en que las cadenze son una creación extempore del intérprete, esto no es así en el caso de Pisendel, al menos no totalmente, ya que existe una preparación previa, no solo de estas cadenże, sino de muchos de los ornamentos que Pisendel usó en sus interpretaciones. Este hecho nos hace preguntarnos por qué Pisendel puso en papel tantos ornamentos, dejando aparentemente de un lado el tradicional carácter improvisado de la ornamentación. 
Lo cierto es que los ejemplos fuera del Schrank II no son demasiados, no muchos intérpretes nos legaron los manuscritos con sus anotaciones personales, si es que estos llegaron a existir. En el caso de los ornamentos que encontramos en otras fuentes significativas, como los ornamentos de Vivaldi transcritos para su alumna Anna Maria contenidas en el conocido «Anna Maria Partbook» ${ }^{57}$, se ha barajado la posibilidad de que muchas de estas improvisaciones escritas tuviesen un origen pedagógico. ${ }^{5}$ En el Schrank II la gran cantidad de estos ornamentos sobre manuscritos vivaldianos (incluyendo autógrafos del propio Vivaldi) y la conocida relación profesor alumno entre Vivaldi y Pisendel parecen apoyar esta teoría, enunciada ya hace tiempo por autores como Kolneder: «[...] el Adagio de un concierto para violín (Hs Dresden Cx 1091) [Concierto para violín y orquesta en do menor, RV 202, Mus.2389-O-122] con disminuciones de Pisendel es muy cercano a los usos de su maestro [Vivaldi] o incluso pudiera haber sido elaborado por él para el uso de su alumno» ${ }^{59}$.

Sin embargo no solo encontramos estos ornamentos en manuscritos de su periodo de estudiante sino que abarcan toda su producción, y sobre todo, están presentes en sus propias composiciones.

Además, para añadir más interés a la situación, existen algunos ejemplos extremos de esta improvisación previamente anotada en la colección de Pisendel. Son muy significativos los manuscritos que Pisendel se preparó para sí mismo para interpretar unos conciertos de Graun, solo han llegado hasta nosotros unos pocos ejemplos, aunque muy probablemente pudiese haber más. Se trata de tres conciertos de Graun: el concierto en la mayor GraunWV C:XIII:91 contenido en los manuscritos Mus.2474-O-21 y Mus.2474-O-21a, el concierto en la mayor para violín GraunWV Cv:XIII:149 contenido en los manuscritos Mus.2474-O-23 y Mus.2474-O-23a y el concierto para violín en mi mayor GraunWV C:XIII:78 contenido en los manuscritos Mus.2474-O-26 y Mus.2474-O-26a. En todos estos conciertos encontramos por un lado un set de partes y la partitura en copia limpia y por otro una parte de violín solista preparada por Pisendel que contiene una versión totalmente ornamentada, en esta ocasión los ornamentos están cuidadosamente copiados como si de una partitura limpia se tratase.

${ }^{57}$ I-Vc, Busta 55.1 (Anna Maria Partbook).

${ }^{58}$ Michael Talbot, «Full of Graces: Anna Maria receives Ornaments from the Hands of Antonio Vivaldi», en Arcangelo Corelli fra mito e realtà storica: nuove prospettive d'indagine musicologica e interdisciplinare nel 350 o anniversario della nascita: atti del Congresso internazionale di studi: Fusignano, 11-14 settembre 2003, ed. por Gregory Barnett, Antonella D’Ovidio y Stefano

${ }^{59}$ [...] the Adagio of a violin concerto (Hs Dresden Cx 1091) with diminutions by Pisendel is very close to the master's usage, if not indeed elaborated by himself for a pupil's usage.

Walter Kolneder, Performance Practices in Vivaldi (Winterthur: Amadeus-Verlag, 1979), 41. 


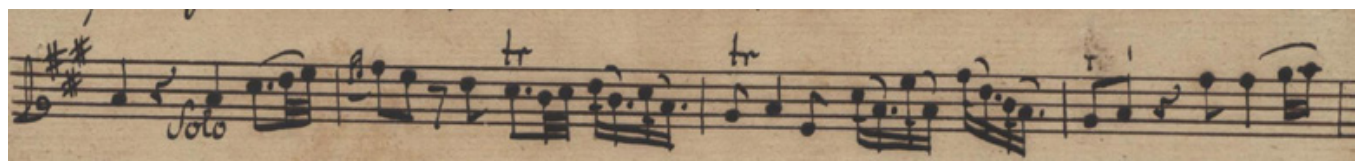

Imagen 14. Graun. Concierto para violín en la mayor GraunWV C:XIII:91. I. Allegro, primera intervención del violín solista (cc. 27-30). Partitura limpia, Mus.2474-O-21a ${ }^{60}$

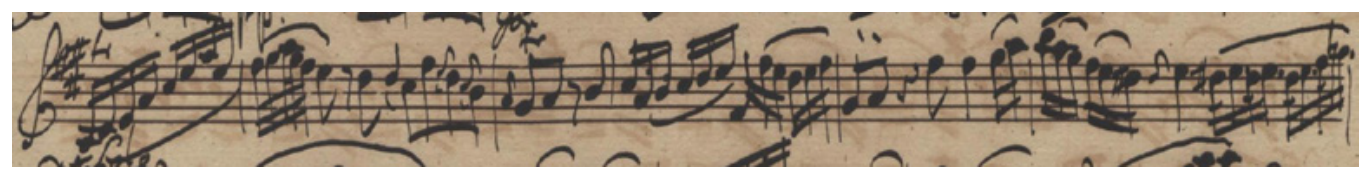

Imagen 15. Graun. Concierto para violín en la mayor GraunWV C:XIII:91. I. Allegro, primera intervención del violín solista (cc. 27-30). Versión ornamentada en la partitura copiada por Pisendel

(Mus.2474-O-21a)

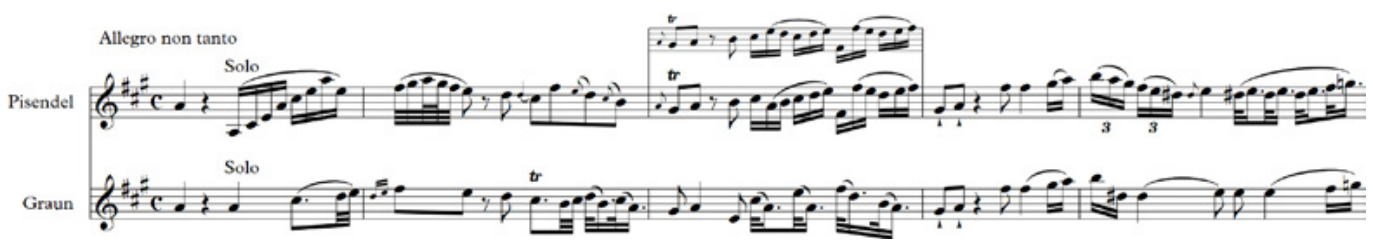

Imagen 16. Graun. Concierto para violín en la mayor GraunWV C:XIII:91. I. Allegro, primera intervención del violín solista (cc. 27-30). Comparación de ambas versiones

Estas versiones ornamentadas contienen todos los elementos característicos de la ornamentación pisendelisana que hemos visto: ornamentos en los movimientos rápidos (desde la primera nota), ricos movimientos lentos e inclusión de cadenze.

Estos ornamentos escritos sobre el papel parecen contradecir a ciertos contemporáneos de Pisendel. Existen fuentes de la época que hacen hincapié en que estos ornamentos no debían escribirse en ningún caso, al menos así lo expresaba Tosi allá por 1723:

Pobre Italia [iLástima, pobres nosotros, pobre nuestra patria!] iQue alguien me explique, por favor! ¡Que alguien me lo explique, por favor! ¿No saben los cantores de hoy dónde se debe hacer la Appoggiature si no se les muestra con el dedo? En mis tiempos lo indicaba inteligencia. ¡Oh eterno reproche a quienes introdujeron por primera vez estas puerilidades infantiles en nuestra nación, que tiene el orgullo de enseñar la mayoría de las artes más bellas, especialmente el canto! ¡Oh, gran

\footnotetext{
${ }^{60}$ Esta parte coincide totalmente con el manuscrito Mus.2474-O-21 que contiene el concierto en partitura.
} 
debilidad la de los que siguen su ejemplo! Oh, injurioso insulto para vosotros cantantes modernos, que sufrís estos documentos de niños. Los extranjeros merecen ser imitados y respetados, pero en aquellas cosas donde sean excelentes. ${ }^{61}$

Esta cita nos sirve para hablar de la improvisación extempore y el estilo italiano. Comencemos con la definición que nos da Rousseau: «El término improvisar es puramente italiano» ${ }^{62}$. El término improvisación está íntimamente ligado a la práctica interpretativa italiana en el barroco. Para ahondar más sobre este tema es muy recomendable el maravilloso artículo de Guido Olivieri del que extraemos esta significativa cita:

La habilidad de improvisación de los italianos, no solo en música y poesía, sino en toda expresión artística, causa asombro y admiración, especialmente entre los viajeros extranjeros. La escritora Anne-Marie Fiquet du Boccage, visitando Roma en 1757, describe una improvisación de Orazio Arrighi Landini: «El Signor Landini tomó su mandolina y, con varias melodías, siguiendo su costumbre, cantó sobre un tema propuesto con versos a menudo extraordinarios. Este talento, desconocido entre nosotros, nos sorprende». ${ }^{63}$

Todo esto hace aún más sorprendente y aparentemente contradictoria la gran cantidad de ornamentos escritos en los manuscritos del Schrank II ya que es evidente la influencia del estilo italiano en Pisendel y está más que suficientemente documentado el dominio de este estilo por su parte. ${ }^{64}$ De hecho, el contacto de Pisendel con la música italiana comenzó desde sus tempranos estudios en

${ }^{61}$ Povera Italia. Ma mi si dica di grazia! Non sanno forse i Cantori d'oggi dì dove vadano fatte le Appoggiature se non gli si mostrano a dito? A mio tempo le indicava l'intelligenza. O eternò biasimo di chi primo introdusse queste puerilità forastiere nella nostra Nazione, che ha il vanto d'insegnar all'altre la maggior parte dell'Arti più belle particolarmente il Canto! O gran debolezza di chi ne siegue l'esempio! O ingiurioso insulto a voi Cantanti moderni, che soffrite documenti da fanciulli. Gli Oltramontani meritano d'esser imitati, e stimati ma in quelle cose però dove sono eccellenti.

Traducción del autor con la inestimable ayuda de Luca Alfonso Rizzello.

Pier Francesco Tosi, Opinioni de' cantori antichi, e moderni o sieno osservazioni sopra il canto figurato (Bolonia: L. della Volpe, 1723), 23.

${ }^{62}$ Le mot improvisar est purement Italien.

Jean Jacques Rousseau, Dictionnaire de Musique (París: Chez la veuve Duchesne, 1768), 255.

${ }^{63}$ L'abilità improvvisativa degli italiani, non solo in musica e posia, ma in ogni espressione artistica, provoca stupore e ammirazione, sopratutto presso i viaggitori stranieri. La scrittrice Anne-Marie Fiquet du Boccage, in visita a Roma nel 1757 descrive un 'imporvvisazione di Oraz̧o Arrighi Landini: «Il Signor Landini prese il suo mandolino e, con melodie varie, siguendo il loro uso, cantò su un soggetto propostogli dei verso spesso straordinari. Questo talento, sconosciuto presso di noi, ci stupisce».

Guido Olivieri, «Naturaleza o Artificio: Riflessioni si Improvvisazione e Virtuosismo Italiani in Francia nel Settecento», en Musical Improvisation in the Baroque Era, ed. por Fulvia Morabito (Turnhout: Brepols, 2019), 290.

${ }^{64}$ Existen muchas referencias en este sentido, aunque los trabajos de Kai Kopp son de los más extensos sobre Pisendel, véase en este caso Köpp, Johann Georg Pisendel..., 106-103 y Kai Köpp, «Johann Georg Pisendel und die von Bach erfundene Viola pomposa (Violoncello piccolo)», en Almanach Ans BACH woche 2005, ed. por L. Thaler y J. Schwinn (Ansbach: Bachwoche Ansbach GmbH, 2005), 53-62. 
Ansbach, donde entró con solo nueve años y recibió clases de Torelli o Pistochi. Precisamente el boloñés Pistochi «que se hizo inmortal por su forma de introducir los ornamamentos» ${ }^{65}$.

Así pues, nos encontramos con que Pisendel, uno de los violinistas más apreciados de su tiempo, elogiado por sus interpretaciones (especialmente en movimientos lentos) y todo un especialista en el estilo italiano, preparaba sobre el papel los ornamentos que más tarde «improvisaría» sobre el escenario. Y no era el único. Aunque no han sobrevivido muchos manuscritos contemporáneos como los de Pisendel, sí que tenemos unos pocos con ornamentaciones de grandes violinistas. Los manuscritos con ornamentos sobre el op. 5 de Corelli elaborados por Dubourg ${ }^{66}$, Festing ${ }^{67}$ o Roman ${ }^{68}$ son buenos ejemplos y muy parecidos a los de Pisendel.

Así mismo podemos fijarnos en las meticulosas transcripciones que realizaron algunos alumnos de grandes maestros, como las ornamentaciones de Geminiani sobre Corelli, ${ }^{69}$ los ornamentos de Vivaldi del libro de Anna Maria ${ }^{70}$ o las ricas ornamentaciones sobre un Adagio de Tartini ${ }^{71}$ y preguntarnos si no fueron copiadas, a tenor de detalle y cuidado con el que fueron transcritas, de los manuscritos de trabajo de los propios autores.

Existen también significativos ejemplos en fuentes más tardías, ${ }^{72}$ como las descripciones de Beethoven improvisando cadenze justo a principios del siglo XIX y encontrar que Beethoven preparó los bocetos para esas cadenze antes de interpretarlas. ${ }^{73}$

${ }^{65}$...who has made bimself inmortal by shewing the way of introducing Graces.

En David Fuller, «Ornamentation», en Companion to Baroque Music, ed. por Julie Anne Sadie (Berkeley: University of California Press, 1998), 418.

${ }^{66}$ Dubourg Corelli's Solos Grac'd by Doburg [sic]., ca. 1721.

${ }^{67}$ GB-Lbl, Add. Ms. 71,244, f. 30. ca. 1736.

${ }^{68}$ S-Sk, Roman Collection, Mss. 61, 97. ca. 1715.

${ }^{69} \mathrm{El}$ manuscrito de la Sonata op. 5 , n. ${ }^{\circ} 9$ de Corelli elaborado por un alumno de Geminiani está perdido, pero fue posteriormente publicado en John Hawkins, A General History of the Science and Practice of Music (Londres: Payne and Son, 1776).

${ }^{70} \mathrm{I}-\mathrm{Vc}$, Busta 55.1.

${ }^{71}$ Un Adagio con los ornamentos de Tartini fue publicado por Cartier en 1798. Para más información sobre esta fuente véase Neal Zaslaw, «Adagio de Mr. Tartini. Varié de Plusier Façons Différentes, Très Utiles aux Personnes qui veulen Apprendre à Faire des Traits sous Chaque Notte de L'harmonie», en Musical Improvisation in the Baroque Era, ed. por Fulvia Moravito (Turnhout: Brepols, 2019), 301-319.

${ }^{72}$ Sobre todo, porque será solamente a partir de la segunda mitad del siglo xIx cuando comience a existir un interés por conservar este tipo de manuscritos tan personales y ligados con el compositor. Se trata de ese cambio de concepción hacia al compositor del que hemos hablado. El estudio de estos manuscritos comenzará precisamente con los bocetos de Beethoven. Para más información véase Sallis, Music Skeetches.

${ }^{73}$ Philip Whitmore, Unpremeditated Art: the Cadenza in the Classical Keyboard Concerto (Oxford Claredon Press, 1991), 202 
La falta de manuscritos de este tipo responde a diversas causas, obviamente vemos su carácter efímero y su inutilidad después de la interpretación, pero es también interesante constatar cómo era parte del negocio el que muchos de estos artistas evitaran en lo posible hacer público «los secretos de su estilo», refiriéndose con esto a la ornamentación como una parte fundamental de ese estilo:

[Los intérpretes] manifiestan, en cierta medida, una preocupación por los negocios, pero al mismo tiempo, dan evidencia de un deseo legítimo, después de todo, de preservar el secreto de su estilo tanto como sea posible. De hecho, la ornamentación expresó, mejor que cualquier otro elemento del arte del intérprete, su propio estilo, su gusto, su personalidad. No siempre les gustó ponerlo al alcance de nadie. ${ }^{74}$

Vemos esto reflejado también algunos manuscritos de la colección de Pisendel y encontramos varios manuscritos donde Pisendel se dedicó a borrar concienzudamente sus anotaciones, seguramente para dejar una copia limpia destinada a su difusión.

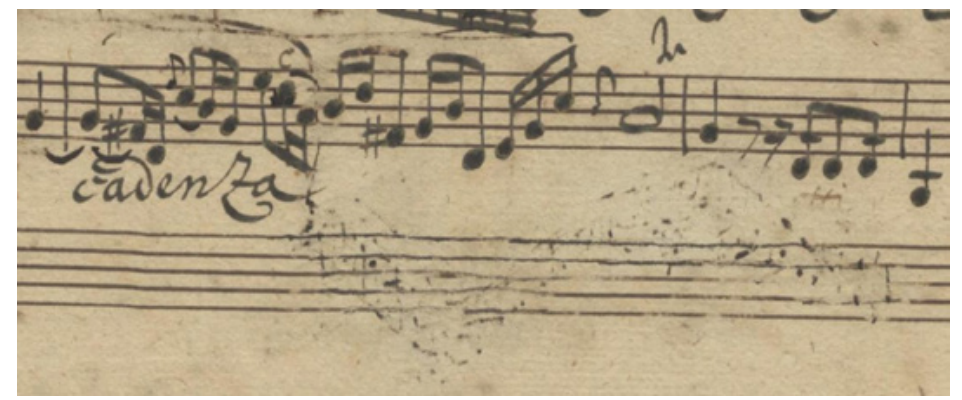

Imagen 17. Christoph Förster, concierto para violín en sol menor. II. Largo, cc. 46-47.

En este ejemplo Förster escribe una cadenza de aproximadamente un compás de duración. Pisendel añade sus propias ideas para aumentar o cambiar esta cadenza. Aún es posible observar los restos de esas ideas que fueron posteriormente borradas

Para entender mejor por qué se escribieron todos estos ornamentos que, teóricamente debían ser improvisados en el momento, hace falta un replanteamiento de lo que la improvisación significaba en aquellos días y darle al término un significado mucho más rico:

${ }^{74}$ They manifest, in a certain measure, a preoccupation with business, but at the same time, they give evidence of a desire legitimate, after all to preserve the secret of their style as much as was feasible. Indeed, ornamentation expressed, better than any other element of the art of the interpreter, his own style, his taste, his personality. He did not always like to put it within the reach of anybody at all.

Marc Pincherle e Isabelle Cazeau, «On the Rights of the Interpreter in the Performance of 17th- and 18thCentury Music», The Musical Quarterly 44, n. 2 (1958): 158. 
Además, lo que revela la historia es que las actividades que incluimos bajo el término «improvisación» comprenden un campo complejo y variado de comportamiento musical, no todos sus elementos tan espontáneos o «en el momento» como podrían parecer. La improvisación suele ser un producto «transfronterizo» creado a partir de la interacción de culturas orales y escritas, o de actividades musicales ensayadas y espontáneas. ${ }^{75}$

Hablar por tanto de ornamentación en el barroco nos trae a un terreno que mezcla la tradición oral con la escrita y la pura improvisación del momento con una concienzuda preparación previa.

Ya en 1602 Caccini nos habla de «una certa sprezzatura del canto» para abogar por el sentido de espontaneidad fingido de los buenos intérpretes. ${ }^{76}$ Alrededor de 1830 el alumno de Chopin, Wilhelm von Lenz, daba vueltas sobre la misma cuestión: «Parece fácil! Chopin solía decir que estos ornamentos “debían sonar como si fuesen improvisados"»".

Esta preocupación por sonar como si se estuviese improvisando, incluso cuando es algo preparado, se enraíza perfectamente con la preocupación de los músicos barrocos de aunar la cultura oral con la cultura escrita de la que nos habla $\operatorname{Pryer}^{78}$, y encuentra numerosísimas referencias en fuentes de la época sobre la imposibilidad de demostrar estos ornamentos de forma escrita. ${ }^{79}$ Esta oralidad en la práctica musical barroca se ve explicada completamente si tenemos en cuenta que estamos en uno de los periodos musicales de la historia donde la retórica se imbrica completamente en todo el acto musical. ${ }^{80}$

Huelga decir que hemos de estudiar la improvisación, la ornamentación y el fenómeno interpretativo en general en su contexto y que las corrientes interpretativas cambian de forma dramática si mudamos de espacios geográficos, temporales o incluso idiosincráticos. Sin embargo en

${ }^{75}$ Moreover, what that history reveals is that the activities we subsume under a single term "improvisation" comprise a complex and varied field of musical behaviour, not all elements of which are quite spontaneus or "in the moment" as they might appear. Improvisation is usually a "cross-border" product from the interaction of both oral and written cultures, or rehearsed and spontaneous musical activity.

Anthony Pryer, «On the Bordelines of Improvisation: Caccini, Monteverdi and the Freedoms of the Performen», en Musical Improvisation in the Baroque Era, ed. por Fulvia Morabito (Turnhout: Brepols, 2019), 149.

${ }^{76}$ Giulio Caccini, «Ai Lectori», Le nuove musiche (Florencia: Marescotti, 1602). Para el término Sprezzatura ver Eduardo Saccone, «Grazia, Sprezzatura, Affettazione in the Courtien», en Castiglione: The Ideal and the Real Renaissance Culture, ed. por Robert Hanning y David Rosand, (New Haven: Yale University Press, 1983), 44-67.

${ }^{77}$ It looks so simple! Chopin used to say of these ornaments that "they should sound as though improvised".

John Rink, "Chopin and Improvisation», en Chopin and His World, ed. por Jonathan D. Bellman y Halina Goldberg (Princeton: Princeton University Press, 2017), 257.

${ }^{78}$ Pryer, «On the Bordelines of Improvisation...», 149.

${ }^{79}$ Para una extensa lista de citas al respecto véase Pincherle y Cazeau, «On the Rights...».

${ }^{80}$ Aunque existen numerosos estudios y escritos sobre el tema me parece especialmente pertiniente citar aquí la obra de Haynes, The End of Early Music. Precisamente Haynes aboga por un cambio en la terminología y usar, en vez de Early Music (música antigua), Rhetorical Music (música retórica). De ahí el título del libro. 
este caso parece claro que es necesario enriquecer el significado de los conceptos de ornamentación, improvisación y composición para este periodo.

En este sentido nos damos cuenta de que la ornamentación es decisiva para completar la composición musical y como vemos es un concepto muy amplio que se disuelve en el propio acto compositivo:

De todos los elementos de la interpretación barroca, la ornamentación, particularmente la que no está indicada por la notación, es la que es más ajena a esas visiones modernas que consideran el texto musical como un registro inmutable de la música. Sin embargo, debería ser una de las consideraciones más importantes en cualquier historia de la música de este período, ya que se refiere al área de cruce entre la interpretación y la composición. ${ }^{81}$

La ornamentación, la propia recomposición de la pieza o su arreglo en la instrumentación se convierten en parte normal de esa «área de cruce entre la interpretación y la composición» ${ }^{82}$. Es posible observar como ornamentación y composición se superponen en ocasiones, diluyendo aún más las fronteras entre los conceptos de ornamentación y composición y expandiendo las relaciones entre ornamentación, improvisación y composición. Esto da un papel mucho más importante a la ornamentación ya que ésta se ve involucrada en todos los niveles de creación e interpretación de una pieza en este periodo.

Es muy revelador que encontremos muchas anotaciones para la ornamentación en los manuscritos de trabajo de Pisendel que se encuentran precisamente aún en una etapa compositiva. Las enmiendas y correcciones de tipo compositivo comparten espacio con anotaciones de tipo interpretativo como digitaciones, ideas para realizar acordes y sobre todo, multitud de anotaciones destinadas a la ornamentación.

Un buen ejemplo lo encontramos en los tres manuscritos que contienen el concierto para violín en mi bemol mayor JunP I.3: el Mus.2421-O-7 contiene la partitura con muchas correcciones y enmiendas de tipo compositivo, el Mus.2421-O-7a contiene las partes que seguramente Pisendel usaría a la hora de interpretar el concierto, con anotaciones para la ornamentación e incluso gran cantidad de digitaciones y ligaduras, y el Mus.2421-O-7b contiene las partes del concierto copiadas a limpio. Si analizamos el manuscrito en partitura Mus.2421-O-7, donde Pisendel aún andaba dando retoques a la

${ }^{81}$ Of all the elements of Baroque performance, ornamentation - particularly that which is not indicated by the notation - is the one which is most alien to those modern views which regard the musical text as an immutable record of the music. However, it should be one of the most important considerations in any history of the music of this period, since it concerns the cross-over area between performance and composition.

John Butt, Music Education and the Art of Performance in the German Baroque (Cambridge: Cambridge University Press, 1994), 121.

${ }^{82}$ Butt, Music Education..., 121. 
composición, encontramos que es el que contiene una gran cantidad ornamentos, mientras que en las partes que fueron usadas para la interpretación solo contienen una guía para estos ornamentos.

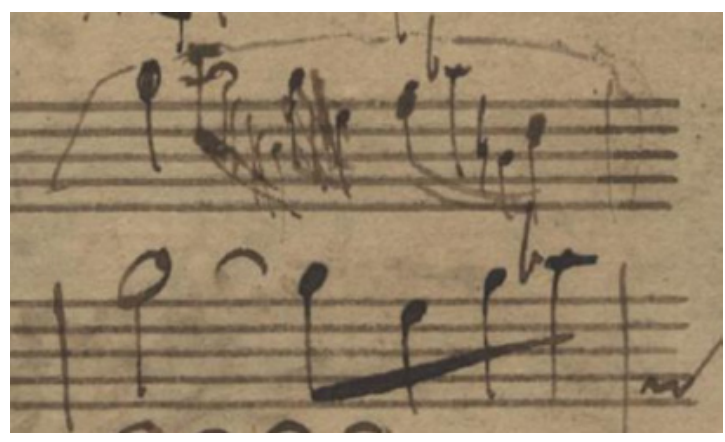

Imagen 18. Pisendel. Concierto para violín en mi bemol mayor JunP I.3. II, Andante, c. 5. Mus.2421-O-7 (partitura de trabajo)

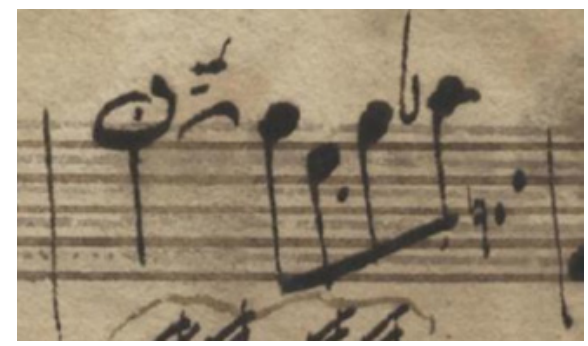

Imagen 19. Pisendel. Concierto para violín en mi bemol mayor JunP I.3. II, Andante, c. 5. Mus.2421-O-7a (partes de trabajo)

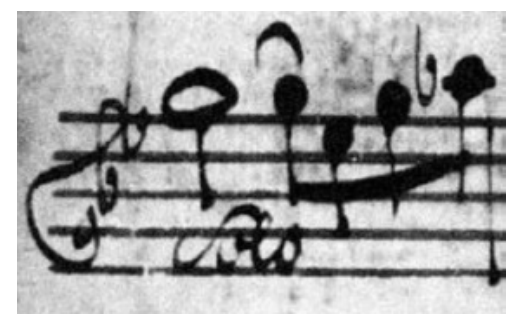

Imagen 20. Pisendel. Concierto para violín en mi bemol mayor JunP I.3. II, Andante, c. 5. Mus.2421-O-7b (copia limpia) 
Este caso encuentra paralelismos no solo en la música para violín y orquesta de Pisendel sino también en su obra de cámara. Otro interesante caso lo encontramos en la sonata en sol mayor ${ }^{83}$ contenida en los manuscritos Mus.2-R-8,37 y Mus.2-R-8,94. Pisendel escribe una gran cantidad de ideas para ornamentar el tema del último movimiento En Rondeau en el manuscrito de trabajo Mus.2-R-8,94 (imagen 21); ninguna de estas ideas aparece en la copia a limpio del Mus.2-R-8,37, donde el tema se repite una y otra vez sin ninguna variación (imagen 22).

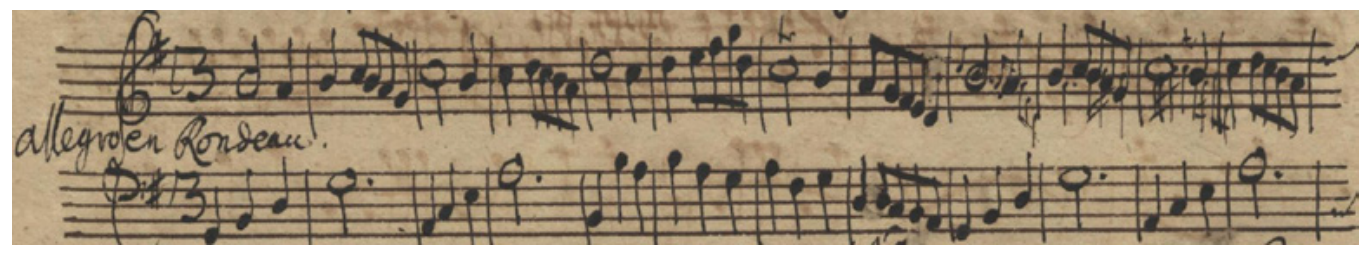

Imagen 21. Doce primeros compases del último movimiento de la sonata en sol mayor para violín contenida en el manuscrito de trabajo Mus.2-R-8,94 con la primera intervención del tema del Allegro en Rondeau y las anotaciones de Pisendel para ornamentar su repetición en el compás 9

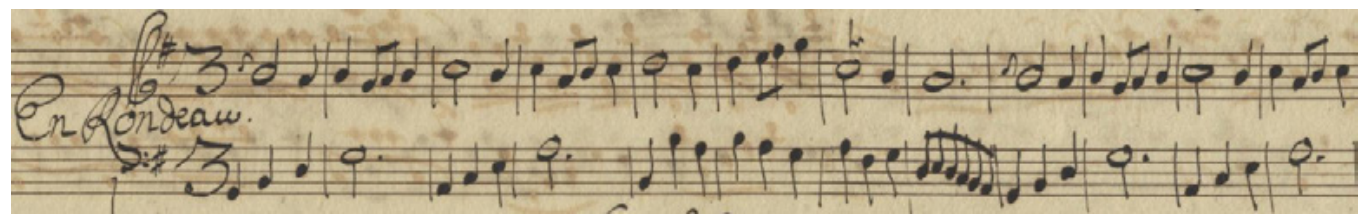

Imagen 22. Doce primeros compases del último movimiento de la sonata en sol mayor para violín. Copia a limpio del Mus.2-R-8,37

A lo largo de todo el manuscrito de trabajo Mus.2-R-8,94 encontramos hasta 9 ideas diferentes para ornamentar el tema del rondeau (imagen 23), aunque este tema solo aparece 8 veces repartidas en 4 refrains.

${ }^{83}$ Esta sonata ha sido recientemente atribuida a Pisendel. Javier Lupiáñez, «Three New Sonatas for violin and Continuo by Johann Georg Pisendel», Ad Parnassum. A Journal on Eighteenth-and Nineteenth-Century Instrumental Music (2020, en prensa). 


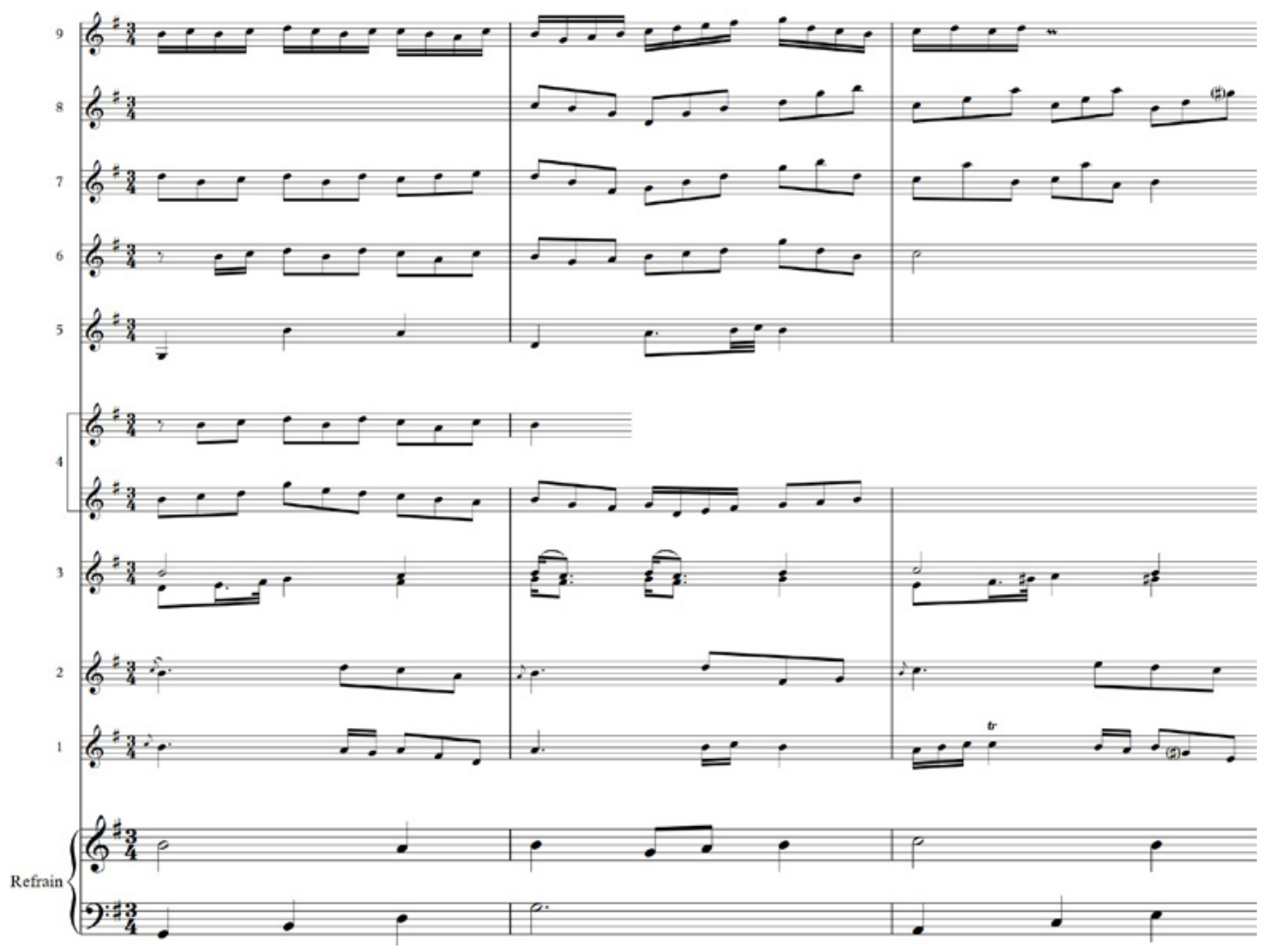

Imagen 23. Sonata en sol mayor (Mus.2-R-8,94). IV. Allegro en Rondeau. Tema (refrain) y sus variaciones

En esta misma sonata encontramos ideas para una cadenza en el manuscrito de trabajo Mus.2-R-8,94 (imagen 24) que no aparece en la versión limpia de la pieza del Mus.2-R-8,37.

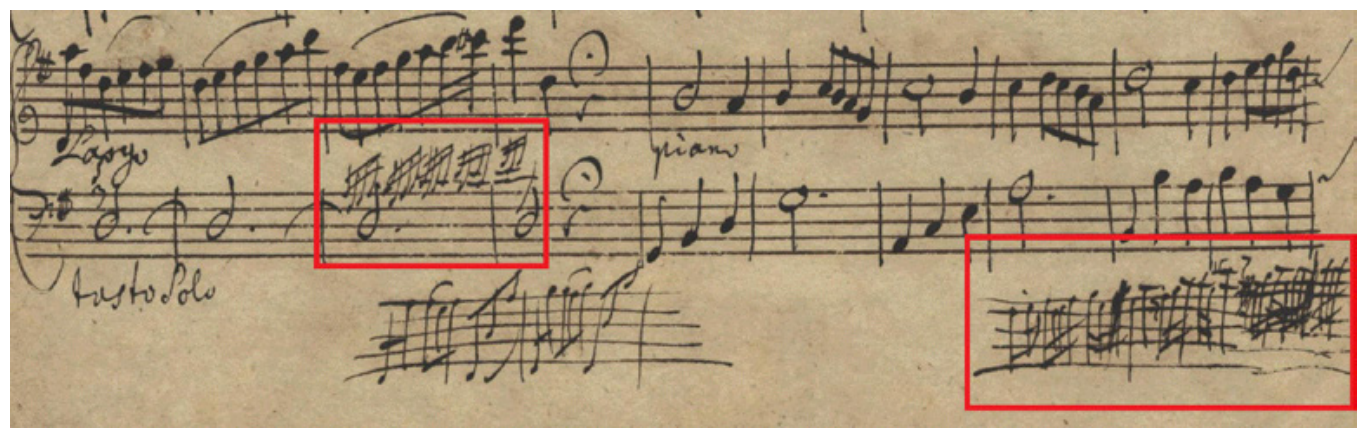




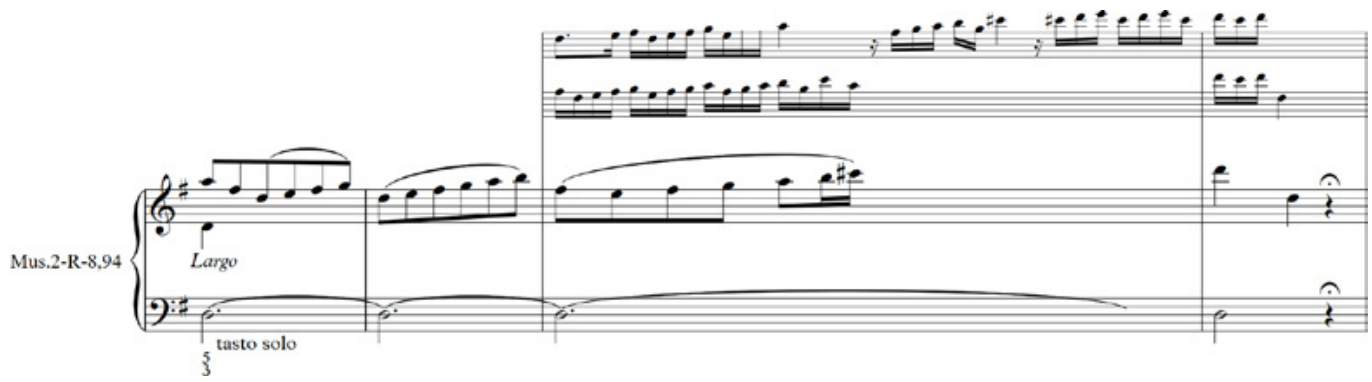

Imagen 24. Sonata en sol mayor. IV. Allegro en Rondeau, cc. 145-158. Cadenza anotada por Pisendel en el Mus.2-R-8,94 y su transcripción

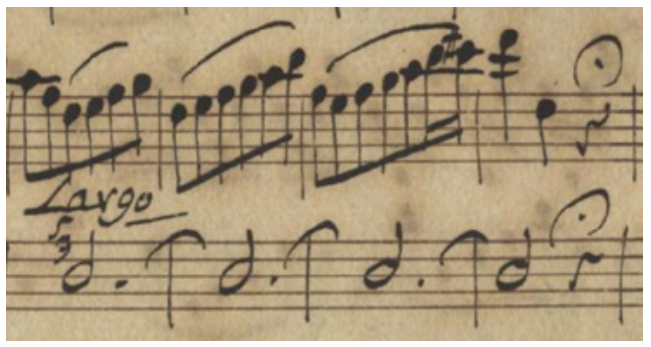

Imagen 25. Sonata en sol mayor. IV. En Rondeau, cc. 145-158. Copia limpia del Mus.2-R-8,37

El hecho de que Pisendel no incluyese estas modificaciones en la última versión de la sonata indica su deliberada intención de dejarlas en manos del intérprete. Algo muy revelador ya que para estas piezas el intérprete seguramente sería solamente él mismo.

\section{Conclusiones}

Antiguamente, la música no se escribía tan escrupulosamente como lo es hoy, y se permitía cierta libertad de interpretación. Esta libertad iba más allá de lo que uno podría pensar, y se parecía mucho a lo que los grandes cantantes italianos dieron ejemplos en los días de Rubini y Malibran. No dudaban en adornar las composiciones, y las reprises eran normales. Las reprises en el sentido de que cuando se cantaba por segunda vez, los ejecutores daban rienda suelta a su propia inspiración. Escuché en mi juventud los últimos ecos de este estilo interpretativo. ${ }^{84}$

${ }^{84}$ Anciently music was not written as scrupulously as it is today, and a certain liberty was permitted to interpretation. This liberty went farther than one would think, resembling much what the great Italian singers furnished examples of in the days of Rubini and Malibran. They did not hesitate to embroider the compositions, and the reprises were widespread. Reprises meant that when the 
Mucho ha cambiado desde los tiempos de Pisendel. Aun así el estudio de las fuentes nos permite acercarnos, aunque solo sea un poco, a los ecos de un estilo interpretativo y una forma de entender la música muy diferente a la actual.

El estudio de los manuscritos de trabajo de Pisendel nos ofrece una visión de la práctica musical personal de uno de los mejores intérpretes de su época; seguramente su forma de actuar y su relación con la música distaría mucho de la de otros músicos contemporáneos, de la misma forma que encontramos gigantescas diferencias entre los grandes intérpretes y músicos de hoy en día, aun cuando estos músicos pertenezcan al mismo campo o se desarrollen en el mismo estilo musical. Aun así, su legado se hace eco de la práctica interpretativa de su época y nos invita a replantearnos de nuevo conceptos básicos.

Vemos cómo el papel del intérprete es de crucial importancia a la hora de terminar el trabajo del compositor. En este proceso la ornamentación juega un papel fundamental en la construcción de la obra musical.

Los manuscritos y las anotaciones de Pisendel respecto a la ornamentación de sus propias obras y de obras ajenas nos muestran un trabajo previo de concienzuda preparación, esto nos lleva a replantearnos el carácter totalmente improvisado de la ornamentación. Esta «improvisación preparada» nos muestra la complejidad intrínseca del arte de la ornamentación e improvisación en este periodo. Esto nos lleva a plantearnos el concepto de improvisación no como actividad completamente extempore sino como un proceso mucho más complejo que requiere, al menos en el caso de intérpretes como Pisendel y de las obras donde nos dejó sus anotaciones, de una preparación previa.

Esto nos conduce a otra de las conclusiones que se destilan al observar muchos de los manuscritos de trabajo de Pisendel, observamos como la composición de la pieza está íntimamente ligada a su interpretación y ésta a la ornamentación. Vemos cómo ideas para la ornamentación surgen durante el proceso compositivo y se omiten a la hora de preparar una copia limpia. La ornamentación es cosa de cada intérprete, algo que lo hace único. Como resultado de esta idiosincrasia resultan lógicas las diferencias que encontramos cuando comparamos las fuentes impresas sobre ornamentación con las fuentes manuscritas destinadas al uso personal o incluso cuando inspeccionemos fuentes de diversos autores.

Volviendo justo al comienzo de nuestro artículo podríamos sacar alguna conclusión más, sobre todo si escuchamos como suenan las palabras de Leonhardt ahora. Y es que la interpretación históricamente informada no es ni mucho menos un ente inamovible e imperturbable. Este cambio hace necesario una revisión

same piece was sung a second time, the executants gave free bridle to their own inspiration. I have heard in my youth the last echoes of this style of performance.

Traducción al inglés de la charla dada por Saint-Saens en el pabellón francés el 1 de junio de 1915 publicada como On the Execution of Music, and principally of ancient Music. A Lecture by M. Camille Saint-Saens Delivered at the "Salon de la Pensie Frangaise" Panama-Pacific International Exposition, San Francisco, June First, Nineteen Hundred \& Fifteen, Done into English with explanatory notes by Henry P. Bowie (San Francisco: The Blair-Murdock Company, 1915). 
periódica de sus ideas, que a veces tarda más tiempo en llegar de lo que nos gustaría. Leonhardt hablaba en esos términos en 1997, ${ }^{85}$ justo un par de años después de que Taruskin publicara Text and $A t^{86}$, donde criticaba el movimiento de la interpretación histórica. Ambas posiciones necesitan ser revisadas y quizá va siendo hora de un análisis profundo y actualizado del estado de la cuestión. De hecho la propia visión de Taruskin sobre la interpretación histórica ha cambiado enormemente desde que escribiera aquellos textos:

Lo cierto es que aquellas versiones que inspiraron sus reflexiones hace cuatro o cinco décadas suenan muy diferentes de las actuales, lo que parece apoyar su tesis de que no estamos más que ante una invención posmoderna en permanente evolución a fin de adaptarse a los gustos actuales: «Las cosas han mejorado extraordinariamente desde los años ochenta, cuando empecé a escribir sobre práctica interpretativa, principalmente porque los intérpretes actuales se escuchan y emulan unos a otros en vez de simplemente aplicar (mal) lecciones aprendidas en los libros». ${ }^{87}$

Quede aquí esta pequeña reflexión personal o este artículo se convertiría en un texto cuanto menos difícil de manejar en cuanto a extensión.

Sirva este artículo para aproximarnos como podamos a algunos de esos aspectos que nos permiten intentar entender mejor el arte musical del siglo XVIII (e indudablemente nuestro propio arte musical), y aunque posiblemente descubriremos que estábamos equivocados, nuestra búsqueda, como la perfección o la felicidad, «es más bien un ideal a perseguir que una meta a alcanzar» ${ }^{88}$.

\section{REFERENCIAS}

Butt, John. Music Education and the Art of Performance in the German Baroque. Cambridge: University Press, 1994.

«Negotiating between work, composer and performer: rewriting the story of notational progress». En Playing with History: The Historical Approach to Musical Performance, 96-122. Cambridge: Cambridge University Press, 2012.

Caccini, Giulio. «Ai Lectori». En Le nuove musiche. Florencia: Giorgio Marescotti, 1602.

Fechner, Manfred. «Improvisationsskizzen und ausnotierte Diminutionen von Johann Georg Pisendel, dargestellt an in Dresden handschriflich überlieferten Konzerten von Johann Friedrich Fasch

${ }^{85}$ Bernard David Sherman, Inside Early Music (Nueva York: Oxford University Press, 1997), 203-204.

${ }^{86}$ Richard Taruskin, Text and Art: Essays on music and performance (Nueva York: Oxford University Press, 1995).

${ }^{87}$ Richard Taruskin entrevistado por Luis Gago, «Malditas preguntas y benditas respuestas», El País, 20 de julio de 2020, https://elpais.com/cultura/2020/07/16/babelia/1594913803 529685.html.

${ }^{88}$ Bruce Haynes menciona a Harry Haskell en el cierre de su libro. Harry Haskell, The early music revival: $A$ history. (Dover, 1988/1996) citado en Bruce Haynes, The End..., 227. 
und Johann Gottlieb Graun». En Zu Fragen der Improvisation in der Instrumentalmusik der ersten Hälfe des 18. Jabrhunderts. Konferenzbericht der 7. Wissenschaflichen Arbeitstagung Blankenburg/Hary, 29. Juni bis 1. Juli 1979, editado por Eitelfriedrich Thom, 35-55. Blankenburg/Harz: Die Kultur- und Forschungsstätte, 1980.

. «Works in Progress. Pisendels Concerti». En Johann Georg Pisendel: Studien zu Leben und Werk; Bericht über das internationale Symposium vom 23. bis 25. Mai 2005 in Dresden, editado por Ortrun Landmann y Hans-Günter Ottenberg, 115-143. Hildesheim: Georg Olms Verlag AG, 2010.

Fuller, David. «Ornamentation». En Companion to Baroque Music, editado por Julie Anne Sadie, 417-434. Berkeley: University of California Press, 1998.

Hawkins, John. A General History of the Science and Practice of Music. Londres: Payne and Son, 1776.

Haynes, Bruce. The End of Early Music. Nueva York: Oxford University Press, 2007.

Hiller, Jahann Adam. Wöchentliche Nachrichten und Anmerkungen, die Musik betreffend, vol. 1. Leipzig: Verlag der Zeitungs-Expedition, 1766.

. Lebensbeschreibungen berühmter Musikgelebrten und Tonkünstler neuerer Zeit. Leipzig: Im Verlage der Dykischen Buchhandlung, 1784.

Jamason, Corey. «The Performer and the Composen». En The Cambridge History of Musical Performance, editado por Colin Lawson y Robin Stowell, 105-134. Cambridge: Cambridge University Press, 2002.

Jones, Gaynor G. «Fürstenau family». En Grove Music Online. Oxford University Press. Acceso el 25 de junio de 2020. http://www.oxfordmusiconline.com/subscriber/article/grove/ music/10402pg3.

Kolneder, Walter. Performance Practices in Vivaldi. Winterthur: Amadeus-Verlag, 1979.

Köpp, Kai. «Das Testament des Dresdner Konzertmeisters Johann Georg Pisendel». En Jahrbuch 1999 der Ständigen Konferenz, Mitteldeutsche Barockmusik, editado por W. Seidel, 59-73. Eisenach: Wagner, 2000.

. «Johann Georg Pisendel und die von Bach erfundene Viola pomposa (Violoncello piccolo)». En Almanach Ans BACH woche 2005, editado por L. Thaler y J. Schwinn, 53-62. Ansbach: Bachwoche Ansbach GmbH, 2005.

- Johann Georg Pisendel (1687-1755) und die Anfange der neuzeitlichen Orchesterleitung. Tutzing: Hans Schneider, 2005.

Lockey, Nicholas Scott. «Second Thoughts, Embellishments and an Orphaned Fragment: Vivaldi’s and Pisendel's Contributions to the Dresden Score of RV 340». Studi vivaldiani 10 (2010): 125-142. 
Lupiáñez, Javier. «Personal Manuscripts as Sources for Ornamentation: the Embellishments of Pisendel in Schrank II, with a Special Focus on Vivaldi». Early Music Performer 46 (2020): 3-19. - «Three New Sonatas for violin and Continuo by Johann Georg Pisendel» Ad Parnassum. A Journal on Eighteenth- and Nineteenth-Century Instrumental Music (2020, en prensa).

Lupiáñez, Javier y Frabrizio Ammetto. «A New Vivaldi Cadenza in an Anonymous Violin Concerto». Studi Vivaldiani 17 (2017): 190-202.

. «Las anotaciones de Pisendel en el Concierto para dos violines RV 507 de Vivaldi: Una ventana abierta a la improvisación en la obra del "Cura Rojo"». En Musical Improvisation in the Baroque Era, editado por Fulvia Morabito, 43-62. Turnhout: Brepols, 2019.

McVeigh, Simon. «Performance in the 'Long Eighteenth Century': an Overview». En The Cambridge History of Musical Performance, editado por Colin Lawson y Robin Stowell, 571-505. Cambridge: Cambridge University Press, 2012.

Mell, Albert y Matthias Wiegandt. «Julius Rietz». En Grove Music Online. Oxford University Press. Acceso el 25 de junio de 2020. http://www.oxfordmusiconline.com/subscriber/article/ grove/music/23451.

Neumann, Frederick. «The Use of Baroque Treatises on Musical Performance». Music \& Letters 48, n. ${ }^{\circ}$ 4 (1967): 315-325.

Olivieri, Guido. «Naturaleza o Artificio: Riflessioni si Improvvisazione e Virtuosismo Italiani in Francia nel Settecento». En Musical Improvisation in the Baroque Era, editado por Fulvia Morabito, 287299. Turnhout: Brepols, 2019.

Pincherle, Marc e Isabelle Cazeau. «On the Rights of the Interpreter in the Performance of 17th- and 18th-Century Music». The Musical Quarterly 44, n. 2 (1958): 145-167.

Pryer, Anthony. «On the Bordelines of Improvisation: Caccini, Monteverdi and the Freedoms of the Performer». En Musical Improvisation in the Baroque Era, editado por Fulvia Morabito, 151-174. Turnhout: Brepols, 2019.

Quantz, Johann Joachim. «Autobiographischer Abriß». En Historisch-Kritische Beyträge zur Aufnahme der Musik. Berlín: Joh. Jacob Schützens sel. Wittwe, 1754.

. On playing the Flute. Traducido por Edward R. Reilly. Londres: Faber and Faber, 1985.

Rasch, Rudolf. «Improvised cadenzas in the cello sonatas Op. 5 by Francesco Geminiani». En Musical Improvisation in the Baroque Era, editado por Fulvia Morabito, 195-222. Turnhout: Brepols, 2019. 
Rink, John. «Chopin and Improvisation». En Chopin and His World, editado por Jonathan D. Bellman y Halina Goldberg, 249-270. Princeton: Princeton University Press, 2017.

Rousseau, Jean Jacques. Dictionnaire de Musique. París: Chez la veuve Duchesne, 1768.

Saccone, Eduardo. «Grazia, Sprezzatura, Affettazione in the Courtien». En Castiglione: The Ideal and the Real Renaissance Culture, editado por Robert Hanning y David Rosand, 45-67. New Haven: Yale University Press, 1983.

Sallis, Friedemann. Music Sketches. Cambridge: Cambridge University Press, 2015.

Schering, Arnold. «Zur instrumentalen Verzierungskunst im 18. Jahrhundert». En Sammelbände der Internationalen Musikgesellschaft. Siebenter Jahrgang 1905-1906, editado por Max Seiffert, 365-385. Leipzig: Breitkopf \& Härtel, 1906.

Sherman, Bernard David. Inside Early Music. Nueva York: Oxford University Press, 1997.

Talbot, Michael. «The Work-Concept and Composer-Centredness». En The Musical Work: Reality or Invention?, editado por Michael Talbot, 168-186. Liverpool: Liverpool University Press, 2000. . «Full of Graces: Anna Maria receives Ornaments from the Hands of Antonio Vivaldi». En Arcangelo Corelli fra mito e realtà storica: nuove prospettive d’indagine musicologica e interdisciplinare nel 350 onniversario della nascita: atti del Congresso internazionale di studi: Fusignano, 11-14 settembre 2003, editado por Gregory Barnett, Antonella D’Ovidio y Stefano La Via, 253-268. Fusigano: Olschki, 2007.

Taruskin, Richard. Text and Art: Essays on music and performance. Nueva York: Oxford University Press, 1995.

Tosi, Pier Francesco. Opinioni de' cantori antichi, e moderni o sieno osservazioni sopra il canto figurato. Bolonia: L. della Volpe, 1723.

Whitmore, Philip. Unpremeditated Art: the Cadenza in the Classical Keyboard Concerto. Oxford: Oxford Claredon Press, 1991.

Zaslaw, Neal. «Adagio de Mr. Tartini. Varié de Plusier Façons Différentes, Très Utiles aux Personnes qui veulen Apprendre à Faire des Traits sous Chaque Notte de L'harmonie». En Musical Improvisation in the Baroque Era, editado por Fulvia Morabito, 301-322. Turnhout: Brepols, 2019.

Zohn, Steven. «Das instrumental Repertoire der dresdner Hofkapelle in den ersten beiden Dritteln des 18. Jahrhunderts: Überlieferung und Notisten Sächsische Landesbibliothek - Staats- und Universitätsbibliothek, Dresden, 23-25 june 2010». Eighteenth Century Music 8, n. ${ }^{\circ} 1$ (2011): 168-170. 